\title{
Feasibility Investigation of Experimental Visualization Techniques to Study Subcooled Boiling Flow
}

\author{
C. E. Estrada-Perez ${ }^{\mathrm{a}, *}$, Junsoo Yoo $^{\mathrm{b}}$, Yassin A Hassan ${ }^{\mathrm{a}, \mathrm{b}, * *}$ \\ ${ }^{a}$ Texas A $8 M$ University, Department of Mechanical Engineering \\ ${ }^{b}$ Texas A $8 M$ University, Department of Nuclear Engineering, \\ 337 Zachry Engineering Building, TAMU 3133, College Station, TX, 77840-3133, USA.
}

\begin{abstract}
The purpose of this study is twofold: 1) to explore the feasible implementation of whole-field visualization techniques such as infrared thermometry, particle tracking velocimetry, and high speed shadowgraphy to study subcooled boiling flow through a vertical square channel with a single heated wall and 2) to provide subcooled boiling flow experimental measurements based on the methodology developed in this work. To fulfill the first objective, a series of sensitivity studies and uncertainty analyses was performed, from which recommendations for the proper implementation of these visualization techniques for the study of twophase flows are given. The purpose of the second objective is to provide reliable information that can be used for the validation of CFD simulations and for the improvement and development of turbulence models under subcooled boiling conditions. Unique in the presented experimental results is the whole-field simultaneous measurement of turbulence characteristics for both the liquid and gas phases and for their interactions. These measurements were made under transitional conditions from a single phase flow region to a two phase boiling flow condition. The presented experimental results provide whole-field, multiscale measurements that can help improve the accuracy of descriptions of the boiling phenomena.
\end{abstract}

\footnotetext{
${ }^{*}$ Corresponding author

**Principal corresponding author, (979)-845-7090

Email addresses: c.estradaperez@gmail.com (C. E. Estrada-Perez), kaks2000@tamu.edu (Junsoo Yoo), y-hassan@tamu.edu (Yassin A Hassan)
}

Preprint submitted to Experiments in Fluids

February 17, 2015 
Keywords: subcooled flow boiling, particle tracking velocimetry, infrared thermometry, liquid turbulence, bubble dynamics.

\section{1. Introduction}

2 Understanding the influence of two-phase flow parameters on liquid turbu-

3 lence plays a key role in developing numerical models to describe the complex

4 behavior found in subcooled flow boiling. Model development depends upon

5 the existence of experimental data that can provide detailed information about

- the liquid and vapor phases at multiple lengths and times scales. Furthermore,

7 it is desirable to quantify the instantaneous and average influences of bubble

$s$ characteristics on the neighboring liquid turbulence. Although multiple experi-

- mental efforts have been directed toward understanding subcooled boiling flow,

10 the complexity of the phenomenon and its high dependence on the experimental conditions have made it difficult to implement whole-field and non-intrusive innovative experimental techniques that can fulfill the multi-scale and statistical demands required for the description of the stochastic nature of subcooled boiling flow are needed. This study explores the scope and limitations of innovative measuring techniques to study subcooled boiling flow. Efforts for the simultaneous measurements of liquid and vapor parameters and their interactions are described, with an emphasis on fulfilling the temporal and spatial demands for both: average and fluctuating quantities. The presented measurements are accompanied with sensitivity studies to account for accuracy and repeatability.

\subsection{Literature Review}

Multiple researchers have made efforts to study subcooled boiling flow experimentally with optical techniques (Abdelmessih et al. 1972, Klausner et al. 1993; Situ et al., 2004 Okawa et al. 2005. Chu et al. 2011). However, only a handful of works have attempted to measure liquid turbulence modifications due to boiling flow parameters (Roy et al., 1997; Lee et al., 2002, Yun et al. 2008, 2010). These experiments use point measurement techniques. Roy et al. 
28 (1997) utilized a two-component laser Doppler velocimetry system to measure both the liquid and boiling bubbles velocities. They also used a dual-sensor fiber optical probe and a constant temperature hot film anemometer to mea1 sure vapor bubble-related quantities and vapor/liquid temperatures. Lee et al. 32 (2002) provided measurements of local void fractions and velocity profiles for 33 both phases. Their measurements were based on a double-sensor conductivity 34 probe for the local void fraction and vapor velocity and a Pitot tube method 35 for the local liquid velocity. Situ et al. (2004) measured the flow structure of 36 subcooled boiling flow in an annulus. They used a double-sensor conductivity 37 probe to measure local void fraction, interfacial area concentration, and inter38 facial velocities. Yun et al. (2008) performed subcooled boiling experiments in 39 a $3 \times 3$ rod bundle. They used a double-sensor conductivity probe for the va40 por phase measurements and a Pitot tube for the local liquid velocity. Rather 41 than presenting their results as local profiles, they presented interpolated 2D 42 maps of liquid velocity, void fraction, interfacial area concentration, interfacial 43 velocity, and bubble Sauter mean diameter for several conditions. Yun et al. 44 (2010) performed subcooled boiling experiments through a concentric annulus. 45 They used a double sensor optical fiber probe for the local measurement of 46 void fraction, bubbles velocities, Sauter mean diameter, and the interfacial area 47 concentration. In separate experiments using a specially designed Pitot probe 48 and a K-type thermocouple, they also measured the local liquid velocity and 49 temperature.

5o Although the above mentioned experiments delivered relevant information 51 on the average two-phase flow parameter characteristics, they do not provide 52 simultaneous measurements of the bubble and liquid characteristics. The tech53 niques used in these experiments depend upon specialized measurements of ei54 ther the gas or the liquid phase parameters, and the obtrusive nature of the 55 probes and/or probe dimensions make measurements close to the wall a chal56 lenge.

57 To overcome some of the limitations inherent in point measurement tech58 niques, full-field visualization techniques have been used on multiple two-phase 
59 flow studies (Hassan et al. 2005, Dominguez-Ontiveros et al., 2006; Ortiz60 Villafuerte and Hassan, 2006, Koyasu et al., 2009). However, there appears 61 to be a scarcity of subcooled boiling experimental studies that can capture in62 stantaneous whole-field measurements. In response to the need for high quality, ${ }_{63}$ reliable experimental information on subcooled boiling flow, Estrada-Perez and ${ }_{64}$ Hassan (2010) performed whole-field particle tracking velocimetry (PTV) ex-

${ }_{65}$ periments in a square vertical channel with a single heated wall. They focussed ${ }_{66}$ on the liquid turbulence modification due to wall heating. Their results con67 firmed the behavior found in previous studies and provided new information 68 due to the full-field nature of the technique. The new provided information has 69 already been used towards the development of a two-phase wall law and for 7o CFD simulation validation (Končar and Matkovič, 2012).

$71 \quad$ Although a large number of studies have been published, subcooled boiling 72 flow is still not completely understood. Due to experimental and technical dif${ }_{73}$ ficulties, most of these studies are often oriented to measure parameters of only 74 one phase, i.e., either liquid or vapor parameters, with experimental approaches 75 limited to either long-term averages such as average void fraction distribution 76 and average velocity profiles or to instantaneous or fast-occurring events like 77 bubble departure frequency, bubble growth rate, etc. Furthermore, there have 78 only been limited attempts to simultaneously capture the full-field wall heat 79 transfer mechanisms and its interaction with the liquid and vapor dynamics so (Theofanous et al. 2002a, Wang and Sefiane, 2012, Gerardi et al. 2010, The${ }_{81}$ ofanous et al. 2002b, Hetsroni et al. 2000, Barber et al., 2011, Golobic et al. 82 2012, Kim et al. 2012.

${ }_{83}$ The present study is a continuation of the experimental endeavor started by 84 Estrada-Perez and Hassan (2010) and has the objective of improving the mea${ }_{85}$ surement reliability when conducting simultaneous PTV, high speed shadowgs6 raphy (HSS) and infrared thermometry (IR-T) for the study of subcooled flow ${ }_{87}$ boiling phenomena. Although these techniques have been previously validated s8 under multiple experimental conditions (Kähler et al. 2012, Khan, 2012, Kim s9 et al. 2012), no attempt has been made to use all these techniques simultane- 
ously to study subcooled boiling flow. Therefore, issues related to the scope and limitations of these techniques have to be addressed before attempting meaningful subcooled boiling measurements. To this end, a series of sensitivity studies and uncertainty analyses were performed, from which recommendations for the proper implementation of visualization techniques for the study of two-phase flows are given. This work also provides subcooled boiling flow experimental measurements which are based on these recommendations.

This paper is organized as follows. Section 2 presents an overview of the experimental facility, instrumentation, and optical arrangements. Section 3 describes the experimental conditions and the unique features of the proposed measurements. Sections 2 and 3 are especially important because the experimental complexity and induced uncertainties are strongly defined by the experimental arrangement and the way in which the experiments are performed. Section 4 shows the measurement issues found and describes the sensitivity studies performed to quantify the influences of each of the addressed issues. Section 5 defines the uncertainties and discusses how the issues found affect the measurements. Section 6 presents the experimental results of subcooled boiling flow experiments after addressing and correcting for the detected uncertainties, and finally, a brief conclusion as well as recommendations are given in Section 7 .

\section{Experimental Setup}

The experimental facility was designed for the visualization of subcooled boiling flow of refrigerant HFE-301 (3M ${ }^{\mathrm{TM}}$ Novec- $\left.7000^{\mathrm{TM}}\right)$ at low system pressures. The facility consisted of a hydraulic loop and a visualization system. Experimental setup details are given in the following subsections.

\subsection{Hydraulic Loop}

The hydraulic loop consisted of an external loop and a test section, both designed to withstand temperatures in excess of $200^{\circ} \mathrm{C}$ and pressures up to $100 \mathrm{psi}(0.7 \mathrm{MPa})$. The external loop provided thermal and hydraulic steadystate conditions. The system excess energy was removed with a small plate 
heat exchanger connected to a chilling system. This allowed us to control the inlet temperature to the test section. Liquid conditions such as volumetric flow rate, temperature, and density were measured by a Coriolis flow meter and controlled by adjusting valves in the test section. The test section was a rectangular channel made of transparent polycarbonate $30.5 \mathrm{~mm}$ long with a cross-sectional area of $10 \times 10 \mathrm{~mm}$.Energy for boiling was provided through a transparent indium tin oxide (ITO) heater with a length of $25.5 \mathrm{~cm}$ and a width of $9 \mathrm{~mm}$ and a maximum working temperature of about $150^{\circ} \mathrm{C}$. The heater was attached to the lateral interior face of the channel (see fig. 1). The electric current to the heater was provided and adjusted by a DC power supply. To reduce ambient heat losses, the test section was covered with an insulation box. Three walls of the insulation box were made of transparent acrylic and the fourth wall was a sapphire window. The insulation box materials were selected to provide visual access for both the infrared camera and the highspeed cameras.The optical saphire window provided almost 95\% transparency to the infrared wavelengths of interest. An unheated length of about $61 \mathrm{~cm}$ was installed (i.e., $L / D_{h} \approx 61$ ) to ensure fully developed flow. To measure the heater wall temperature, a mid-wave infrared camera $(3-5 \mu \mathrm{m})$ was used. To provide validation for the infrared camera measurements, K-type thermocouples were attached to the external face of the heater. The test section fluid inlet $\left(T_{i n}\right)$ and outlet $\left(T_{\text {out }}\right)$ temperatures were measured with two fast response T-type thermocouples. Ambient temperature $\left(T_{a m b}\right)$ fluctuations were measured with J-type thermocouples. Figure 1 shows the schematics and dimensions of the test section. To account for the pressure drop throughout the test section, two differential pressure transducers were used. Also, the gage pressure was measured at the entrance $\left(P_{\text {in }}\right)$ and at the outlet $\left(P_{\text {out }}\right)$ of the test section. The parameter uncertainties are provided in Table 1.

[Figure 1 about here.]

[Table 1 about here.] 
176 177

\subsection{Visualization System}

The visualization system consisted of numerous parts. They were three high-speed, high-resolution cameras: two for shadowgraphy experiments and one for the simultaneous PTV-shadowgraphy technique; a high-speed, highpower laser; a high-speed infrared camera; a fast response light emitting diode (LED) system; mirrors; translational stages; lenses; optical filters; particle flow tracers; and a laser power meter. The flow tracers were fluorescent particles with a density range between 1.39 to $1.41 \mathrm{~g} / \mathrm{cm}$ with an average particle diameter of $5 \mu \mathrm{m}$. Illumination for PTV experiments was provided by a Nd:YLF dualhead laser system (ESI, New Wave Research) which offers a nominal energy of $10 \mathrm{~mJ}$ of $527-\mathrm{nm}$ light at $1000 \mathrm{~Hz}$. Each laser heads can provide up to 5000 pulses per second, allowing PTV measurements up to 10,000 frames per second with lower energies than the nominal. Two optical mirrors and two concave-convex lenses were used to convert the small circular beam from the laser, into a thin sheet of light. The laser light sheet was positioned parallel to the camera focal area. The LED system is externally synchronized to be used as the shadowgraphy illumination. The cameras, mirrors, and lenses were mounted on translational stages in order to have the capability of measuring at different locations within the test section. Figure 2 shows the schematics of the visualization system. The high speed cameras, the laser, and the LED illumination system were synchronized in such a way as to be able to obtain quasi-simultaneous PTV and shadowgraphy measurements.

[Figure 2 about here.]

\subsubsection{Temporal Multi-Scale Approach}

The configuration shown in Figure 2 was designed to allow multi-orientation and multi-scale measurements. The high-speed PTV/shadowgraphy camera was arranged to provide semi-simultaneous measurements of PTV and shadowgraphy. This was achieved by synchronizing the camera shutter with alternating pulses of laser and LED illumination. From the temporal multi-scale point of view, the $\mathrm{PTV} /$ shadowgraphy camera can be configured to run in continuous 
$\mathbf{1 7 8}$ or in straddling timing modes. Continuous mode provides a fixed time inter-

205 206 phases.

[Figure 3 about here.]

\subsection{Spatial Multi-Scale Approach}

To achieve spatial multi-scale measurements, the PTV/shadowgraphy camera was equipped with a telecentric lens which allowed the selection of two different visualization modes, high and medium magnification modes, which corresponded to resolutions of 4.82 and $17.5 \mu \mathrm{m} /$ pixel, respectively. The two shadowgraphy cameras were configured to have different visualization modes: One camera provided high magnification while the other camera was set at low magnification. The low magnification corresponded to a resolution of $187 \mu \mathrm{m} /$ pixel. Focus will be given to the results from the medium-magnification PTV/shadowgraphy camera, which provided simultaneous information from both liquid and vapor 
212

213

214

215

216

217

218

219

220

221

222

223

224

225

226

227

228

229

230

231

232

The experimental setup described above allows for a set of unique experimental features relevant for two-phase flow measurements. These are summarized next.

Simultaneous whole-field measurements of liquid and bubbles parameters:

- The simultaneous application of PTV and shadowgraphy techniques provided measurements of the liquid and bubbles velocities along with measurements of parameters that describe bubble dynamics. This methodology simplifies quantification of the local void influence on the liquid turbulence.

- Improved liquid velocity measurements can be achieved by using the shadowgraphy information to remove spurious vectors found within the domain occupied by the boiling bubbles, i.e., liquid velocity vectors should not exist within the bubble volume.

- Fluorescent seedings and optical filters helped reduce bubble-induced laser light reflections. It is known that bubble-induced reflections accounts for a large percentage of undesired noise in the PTV images.

The use of a relatively long and transparent heater for boiling:

- The heater's length provided enough heating distance to obtain thermally and hydraulically fully developed flows within the test section.

- The heater transparency allowed its use for either, visualization or illumination access.

Spatial and temporal multi-scales measurements:

- The availability of visual access from different camera perspectives allows the quantification of bubble parameters with respect to the $x-y$ and $x-z$ planes, while at the same time providing near-wall liquid velocity measurements in the $x-y$ plane. 
- The spatial multi-scale visualization configuration allows for liquid turbulence measurements to be resolved for both the largest and smallest scales within the test section.

- The spatial multi-scale visualization allowed resolution of detailed nearwall liquid velocity measurements and three different scales to study the bubble dynamics during the lifetime of the bubbles.

- The continuous and straddling timing modes allowed for temporal multiscale measurements which helped resolve the fast-happening instantaneous parameters while providing also a venue for resolving the long-term average measurements. This capability proved to be specially important for the average and instantaneous bubbles dynamics measurements.

Infrared thermometry:

- Infrared thermometry allowed for measurements of 2D temperature fields of the heater wall. Only average temperature fields were considered. The infrared measurements were useful to ensure experimental replicability, because deviations from the normal boiling behavior were identified as outliers in the measured temperature fields.

Analysis of a single nucleation site:

- By placing the measurement area in the vicinity of a single nucleation site, it was possible to measure the flow development from a single-phase heated condition in its transition to a two-phase bubbly flow condition; i.e., measurements for both upstream and downstream of the nucleation site, covering the complete bubble life-cycle were available.

- Measuring a developing bubble through its growth process facilitated the understanding of the influences between bubble parameters and liquid turbulence, such as with the relationship of bubble diameters on the liquid velocity.

Improved experimental practices: 


\section{3. Experiments}

266

\section{Addressed Issues}

- An experimental protocol was developed to reduce the influence of noncondensable gases, laser-induced heat, optical distortions due to temperature gradients, and heater aging. This protocol helped to achieve reasonable replicability and reduced uncertainty.

\subsection{PTV-IR-Shadowgraphy experiments}

The PTV/Shadowgraphy camera measurement area was located at about $12.3 \mathrm{~cm}$ from the test section inlet. Simultaneous PTV and shadowgraphy measurements were performed at this position. The high-speed camera was set to 4000 frames/s, with an exposure time of $250 \mu \mathrm{s}$. The relatively large exposure rate was selected as to allow enough light to reach the camera sensor and achieve satisfactory shadowgraphs of the bubbles. The time between the PTV and the shadowgraphy pairs was $\Delta t_{P T V}=1 / 2000$. This timing allows to acquire one frame of PIV immediately followed by one frame of shadowgraphy. For the medium magnification experiments, each acquired image consisted of $640 \times 952$ pixels and a resolution of $17.5 \mu \mathrm{m} /$ pixel. Only one experimental condition was considered, with a Reynolds number of $\operatorname{Re}=4400$. For this Reynolds number, a heat flux of $q^{\prime \prime}=13.5 \mathrm{~kW} / \mathrm{m}^{2}$ was selected and a constant inlet temperature of about $15^{\circ} \mathrm{C}$ was maintained. This experimental condition allowed for a stable nucleation site behavior, however, as can be seen later, nucleation intermittency was found to be a natural behavior at this conditions. The heater wall temperature was measured with the infrared camera. The infrared camera magnification allowed temperature measurements of the whole length of the test section with a resolution of $265 \mu \mathrm{m} /$ pixel. Inlet and outlet fluid temperatures were also measured by means of T-type hypodermic thermocouples.

As discussed previously, there exist a vast collection of works which focus on subcooled boiling. However, there are not many subcooled boiling experimental studies utilizing visualization techniques to study simultaneously the behavior 
of both phases and their influence on the heater wall temperature. The reason of the scarcity of visualization studies dealing with subcooled boiling studies may be due to the experimental inherent complexity and the relative incompatibility between these visualization measurement techniques and the boiling phenomena of interest. For example, if not properly addressed, the visualization instrumentation may induce perturbations into an otherwise statistically stable boiling system. In this section are summarized the sensitivity analysis of experimental issues not previously addressed in other studies. These issues are classified in three categories a) visualization experimental issues, b) error sources due to the boiling phenomena, and c) data analysis issues. The methodology to quantify and minimize the experimental uncertainties are presented in the next sections.

\subsection{Visualization Experimental Issues}

\subsubsection{Illumination Heat Sources}

Most of the visualization experimental techniques ${ }^{1}$ share the characteristics of utilizing specialized illumination sources. These illumination sources will depend on the targeted parameters of interest. The 2D PTV technique utilizes a high energy 2D laser light sheet for liquid velocity measurements, while the shadowgraphy technique utilizes volumetric illumination, such as an halogen or LED lamp, to generate shadows of the objects under investigation. These illumination sources produce large amounts of heat, and since in this study, the PTV and the shadowgraphy techniques were utilized simultaneously, the impact of the illumination instrumentation induced heat on the boiling system was addressed and quantified. It is important to mention that similar studies were performed before (Estrada-Perez and Hassan (2010) $)$, however, previous studies limited their analysis to understand the impact of illumination heat sources on the wall temperature by means of wall-thermocouples. In the present study the multi-view, multi-scale visualization and the added advantage of a high-speed infrared camera allowed to capture the effect of the illumination heat sources

${ }^{1}$ Although IR thermoetry technique was also used in this study, it was not considered in this section because IR themometry do not require an external illumination source. 
318 in both, the bubbles dynamics and the heater wall temperature. The present sensitivity studies are summarized in the next sections.

320

321

322

323

324

325

326

327

328

329

\subsubsection{Selected timing modes (Straddling vs Continuous)}

The illumination system was configured to run in continuous and straddling modes (see section 2) with two illumination sources; a high energy laser and an LED lamp. A sensitivity analysis was performed to quantify the influence of these illumination sources and to explore ways to minimize non-desirable effects. Four tests were considered: a) LED illumination and continuous timing mode, b) LED illumination and straddling timing mode, c) LED+laser illumination and Continuous timing mode, and d) LED+laser illumination and Straddling timing mode. The effect of the illumination timing modes was studied from the measurement results of bubbles dynamics and from the Infrared heater wall temperature measurements.

\subsubsection{Effect on Bubbles Dynamics}

For this analysis, we focused on the bubbles dynamics behavior. Figure 4 shows the comparison of the near-nucleation site bubble dynamics between the different illumination and timing modes. Figure 4 shows a two dimensional plot of the bubbles centroid location for each case ( $\mathrm{X}$ is the axial direction, while $\mathrm{Y}$ is the wall-normal direction). For these conditions the bubbles rarely detached from the wall, therefore the bubbles centroid location gives a very good indication of the bubble radius. It is noticeable that for almost all cases the bubbles paths followed a similar trajectory. However, the Laser+LED Continuous case showed to be different from the other test cases. The added amount of heat by the laser running in Continuous timing mode, produced bubbles even upstream the nucleation site under investigation. These laser-induce bubbles, while sliding through the wall, travel on top of the nucleation site causing it to deactivate or making it intermittent. Of special interest are the comparisons between LED Straddling and Laser+LED Straddling cases, in both cases the bubbles paths seem virtually the same. This gives a clear indication that the laser induced effects are minimal when the Straddling timing mode is used. It is important 
348 to note that although the LED Continuous case presents very similar bubble

349 behaviors, the bubbles most probable paths are not captured. This may be

363

364

374 375 due because in the Continuous timing mode, fewer independent samples are acquired hindering the overall statistics of the measurements, i.e., a single bubble is captured in multiple frames, therefore reducing the sampling number. With the Continuous timing mode, outliers bubbles may impact more the statistics.

[Figure 4 about here.]

Figure 5 compares the estimated bubbles diameters measured in each of the experimental modes. From the box plots is noticeable that even when the average bubble diameter is about the same $(0.52 \mathrm{~mm})$ much more outliers are found in the Laser+LED Continuous mode. From these plots can be concluded that if the straddling timing mode is selected it can be ensured similar behavior in repeated experiments. The shadow illumination continuous mode provides similar characteristics but with a higher number of outliers.

[Figure 5 about here.]

From this analysis it was concluded that as long as the straddling timing mode is used, non-desirable influences from the illumination sources are considered to be negligible small.

\subsubsection{Effect on the heater wall temperature.}

As mentioned previously, the effects of the illumination sources and timing modes have a significant effect on the bubbles dynamics. In a similar way, illumination sources also affect the heater wall temperature, and when considering that boiling bubbles dynamics are intimately linked to heater wall heat transfer, these effects become more evident. Two mechanisms were found on which illumination sources may change the heater wall temperatures, the first mechanism is the direct energy deposition from the illumination sources to the heater wall.

The direct energy deposition may be absorbed uniformly by the whole heater, as in the case of halogen and LED lamps for shadowgraphy measurements, or 
it may be absorbed only in particular locations, such as in the case of PTV, on which the laser light sheet was restricted to a line of $0.8 \mathrm{~mm}$ thickness impinging on the heater wall. From infrared measurements, the direct deposition of energy from illumination sources appeared to be negligibly small, and was barely detected when single phase experiments were performed. The second mechanism were noticeable only in the boiling experiments, and significantly changed the heater wall temperature. The wall temperature distorting mechanism is indirect in nature and initiate due to small perturbations of the heater wall temperature. The small temperature perturbations lead to modification of the bubbles dynamics in the form of illumination induced boiling. The illumination induced boiling brought about significant changes in the heater wall temperature, as can be seen in figure 6. Figure 6 shows the results of a sensitivity study on which four cases were considered: a) No illumination, b) LED illumination and Straddling timing mode, c) LED+laser illumination and Straddling timing mode and d) LED+laser illumination and Continuous timing mode. From these results is clear that the LED+laser illumination with continuous timing mode produced significant changes when comparing to the no illumination case. The temperature changes were not so significant in the single-phase region, there seems to be a constant temperature increase up until the vicinity of the nucleation site (about $130 \mathrm{~mm}$ from the inlet electrode). The laser induce bubbles acted as a heat sink in the nucleation site region, making it intermittent. The nucleation site intermittency reduced the heat removal due to a reduced bubble frequency, which is translated in a temperature increase downstream of the nucleation site as seen in figure 6. All other illumination cases on which the laser light illumination in a continuous timing mode were not used, provided reasonable and repeatable results. 
403 4.2. Error Sources of Subcooled Boiling

404 4.2.1. Thermally induced optical distortions

405 Thermally induced optical distortions (TOD) in subcooled boiling systems

406 are typically found nearby interfaces on which large temperature gradients oc-

407 cur. Of special interest on subcooled boiling are the heater wall-liquid interface

408 and the boiling bubbles-liquid interfaces. The TOD phenomenon is specially rel-

409 evant on organic fluids on which the refractive index is strongly dependent on

410 the local fluid temperature (Elsinga et al. $(2005))$, as is the case for the present

411 study. The working fluid selected for the current experiments is relatively new,

412 and the temperature dependance of the refractive index is unknown. Therefore

413 an experimental characterization was performed to test the influence of refrac-

414 tive index changes on the optical "performance" of the visualization techniques.

415 From the analysis, it was found that the thermally induced optical distortions

416 have three main effects, one bias effect, and two random effects: one due to den-

417 sity changes and the other due to optical distortions produced by the boiling

418 bubbles. These effects can be accounted to be included part of the bias $\left(\beta_{T O D}\right)$

419 and random uncertainty $\left(\sigma_{T O D}\right)$ of the PTV centroid location algorithm. The

${ }^{420}$ quantification of these effects were performed in situ, with the following process:

${ }^{421}$ A calibration grid with circular patterns was located coinciding with the focal

422 plane of the PTV/Shadowgraphy camera. In this configuration, the grid pattern

${ }^{423}$ is normal to the heater wall, having one edge in the near wall region (right side

${ }^{424}$ of figure 7) and the other edge in the bulk liquid region. This grid was gen-

${ }_{425}$ erated with a distance of $0.5 \mathrm{~mm}$ between each circular pattern centroid. The

426 flow loop was ran at the conditions of interest, namely at a Reynolds number of

${ }_{427} R e=4400$, a heat flux range from 0 to $13.5 \mathrm{~kW} / \mathrm{m}^{-2}$ and a subcooling level of

${ }_{428} \Delta T_{s u b}=15^{\circ} \mathrm{C}$. The PIV/Shadowgraphy camera frame rate was set to $2000 \mathrm{fps}$,

429 the same frame rate as the one used for the "continuous" timing condition (see

430 section 2.2.1. The experimental conditions of the uncertainty analysis emulate

431 exactly the boiling experimental conditions, with the exception of the camera

432 focal plane location. In this analysis, the camera was focused on the calibration

${ }^{433}$ grid, rather than on the nucleation site. The calibration grid was located on 
434 the outer face of the channel, at about $6.5 \mathrm{~mm}$ from the nucleation site (the

435

436

437

438

439

440

441

442

443

444

445

446

447

448

449

450

451

452

453

455 Random uncertainty due to thermally induced optical distortions ( $\left.\sigma_{T O D}\right)$. As

nucleation site was located at about $3.5 \mathrm{~mm}$ from the inner wall). This location was selected as to maximize the illumination light path length through the medium with variable refractive index (the heated refrigerant) on its travel from the target (the calibration grid) to the camera sensor.

Shadowgraphy pictures of the calibration grid were acquired at five different heat fluxes. 1000 images were stored per condition. The acquired calibration grid images were pre-processed to match the requirements of the PTV centroid location algorithm, which receives as input an image with white objects embedded in a black background. The PTV centroid location algorithm was applied for all images within each set. Figure 7 shows the centroid location process applied to the calibration grid. Figure 7 (a) shows the inverted (or negative) version of the original calibration grid image which is the input of the PTV centroid algorithm, figure 7 (b) shows the output of the PTV centroid location algorithm. The PTV centroid location algorithm is based on a particle mask correlation method (Takehara and Etoh (1999)), on which every pixel is correlated to an "ideal" object image, in this case, a white circle embedded in a black background. The blue pixels in the output image indicate pixels near the vicinity of the centroid, while the light yellow pixels indicate the centroid position itself.

[Figure 7 about here.] mentioned previously, the random component of the thermally induced optical distortion can be divided in two, those due to density fluctuations without phase change $\left(\sigma_{T O D_{\rho}}\right)$ and those due to the bubbles passing through the measurement region $\left(\sigma_{T O D_{b}}\right)$, therefore, the total TOD random uncertainty can be expressed as $\sigma_{T O D}=\sigma_{T O D_{\rho}}+\sigma_{T O D_{b}}$. These random components of the thermally induced optical distortions were estimated from the calibration grid images. The random distortions without phase change $\left(\sigma_{T O D_{\rho}}\right)$ showed a concomitant behavior with the heat flux. As expected, these distortions showed to be larger 
464 for the higher heat flux cases, and for the regions within the vicinity of the

485

486 heated wall. The effect of the random distortions on the calibration grid images appeared as image deforming patches which moved in the flow direction. These patches varied in length and width affecting differently the appearance of the grid images. Ultimately, the grid points location estimation was randomly changed by these patches. To clarify this effect figure 8 shows the centroid location estimation of the calibration grid points. These results are shown for the non-heated and boiling conditions $\left(q^{\prime \prime}=0\right.$ and $q^{\prime \prime}=13.5 \mathrm{~kW} / \mathrm{m}^{2}$ respectively). It is important to mention that the boiling bubbles are outside of the camera focal plane, reducing in this way their optical distortion influence, therefore it is safe to say that the present analysis quantifies only the uncertainty due to the effect of the liquid density changes. Figure 8 shows the calibration grid points centroid location estimation for all 1000 images for both, the unheated and boiling conditions. It is clear that the centroid location fluctuations are larger for the boiling condition, which is represented by the black crosses, while the red crosses represent the centroid location estimation on the unheated condition. It is important to mention that although no variations on the grid points centroid locations were expected for the unheated case, small variations were found of about 0.1 pixels. These variations are mostly attributed to the LED illumination fluctuations and due to the fact that the PTV algorithm will produce larger uncertainties for centroid estimation of big objects (Estrada-Perez and Hassan (2010)). Therefore we define here a random error inherent to the PTV algorithm for big objects as $\sigma_{P T V}=0.1$ pixels.

\section{[Figure 8 about here.]}

Further conclusions may be drawn from the wall-normal RMS profiles of the centroid location estimation. These profiles are shown in figure 9, The centroid location RMS of the boiling case is a function of the wall-normal position, while the single non-heated case shows to be position independent. The boiling case showed to have a maximum RMS of about 0.6 pixels on the near-wall region, decreasing asymptotically for regions far from the heated wall towards a value 
494 of about 0.15 pixels. Noting that the boiling asymptotic RMS value is slightly

495 different to 0.1 (which is representative of the single phase case), lead us to

496 conclude that the influence of the thermally induced optical distortions affect

497 the whole measurement view and need to be accounted for. Another conclusion

498 from this analysis was the fact that the centroid location random uncertainties

499 due to thermally induced optical distortions has an equal effect on both, the

500 wall-normal and the wall-parallel directions.

501 From figure 9 we can obtain a maximum value for $\sigma_{T O D_{\rho}} \approx 0.5$ pixels (con-

502 sidering that about 0.1 pixels is attributed to the algorithm uncertainty at this

503

504

505

506

507

508

509

510

511

512

513

514

515

516

517

518

519

520

521

522 523 heat flux is readily available.
[Figure 9 about here.]

Bias uncertainty due to thermally induced optical distortions $\left(\beta_{T O D}\right)$.. An apparent displacement of the calibration grid points was observed as a consequence of heat flux increments. This displacement was uniformly distributed through the whole image with a wall-normal preferential direction. The overall trend was that for every increment on heat flux, the whole image seemed to shift towards the direction where the heated wall was located. This effect was so unexpected and large, that it was wrongly thought that the camera or the channel were displaced in some way. The apparent displacement can be observed, when comparing the grid points centroid estimation of the boiling and unheated conditions from figure 8. To clarify this effect, figure 10 shows a single row of grid points for the five different heat fluxes considered for this analysis. This apparent displacement $\left(\Delta y_{o}\right)$ is stable once a steady state condition is reached, after which an estimation of $\beta_{T O D}$ can be easily obtained and a correction for each 
525 Random uncertainty due to the bubbles passing through the measurement region

$526\left(\sigma_{T O D_{b}}\right)$. From all the thermally induced optical distorting effects, the one that

${ }_{527}$ brought about the largest uncertainties was the boiling bubbles effects. To quan-

528 tify the effect of the boiling bubbles, a calibration grid was located exactly on

${ }_{529}$ top of the nucleation site. The camera focal plane was located in the calibra-

${ }_{530}$ tion grid position so as to have the calibration grid on focus. From the camera

${ }_{531}$ perspective, the boiling bubbles passed in front and behind of the calibration

${ }_{532}$ grid. Only the bubbles passing in front of the calibration grid produced optical

${ }_{533}$ distortions. To clarify this procedure, figure 11. a) shows the optical distortions

${ }_{534}$ that a seemingly transparent boiling bubble produce on its path in front of four

535 calibration grid points. The boiling bubble is sliding along the heater moving

${ }_{536} \mathrm{up}$ in the direction of the flow. The frames at times $t=0.0$ and $t=4.0 \mathrm{~ms}$

537 shows examples when the influence of the bubble was minimal. It is clear that

${ }_{538}$ at those particular times the centroid estimation of the four points were accu-

539 rately captured. However at different times, the bubbles domain was nearby of

540 the measured area, and the bubbles influence was clear. The curvature of the

${ }_{541}$ bubbles interface produce a lens-like effect which produced the optical aberra-

${ }_{542}$ tions of the calibration grid points.

544 A more quantitative approach may be observed on figure 12 where the 545 black dots represent the centroid location estimation from a row of the calibra546 tion grid. The bubbles influence is confined to near-wall regions, on which the 547 boiling bubbles are located. The bubbles induced uncertainties showed a similar ${ }_{548}$ behavior as the liquid density fluctuation uncertainties in the sense that there ${ }^{549}$ is not an influence preferential direction. The centroid location of grid points 550 varied within a range of 15 pixels which in the context of PIV/PTV is unaccept${ }_{551}$ able. Therefore an approach to reduce this influence is strongly recommended.

552 In this work we removed all the bubbles induced optical distortions by using 
553 the bubbles images as masks to remove this undesired effect. The process of

$\mathbf{5 5 4}$ bubbles masking was facilitated due to the fact that we performed simultaneous

555 PIV and Shadowgraphy visualizations, this meant that we can use the bubbles

556 information from the shadowgraphs to remove the unreliable particles found

557 within the domain of the bubbles. Figure 11,b) shows the bubbles masking

558 procedure to reduce the effect of the bubbles passing. In this case the bubbles

559 body is considered to be no longer transparent, therefore the lensing-effect is

560 totally removed. However, bubbles influence still prevails in the vicinity of the

${ }_{561}$ bubbles. In these regions, large density changes are still producing considerably

562 large effect. However it has to be recalled that in the PIV/PTV experiment,

563 the objects to be tracked are tinny particles that are following the liquid flow.

564 Both of these characteristics (small size and moving with the flow) will reduce

565 greatly the bubbles influence. The present analysis is quite conservative, and it

566 is expected that both, the density fluctuations and bubbles influences will af-

${ }_{567}$ fect the PIV/PTV particle tracers centroid location in a reduced manner. The

568 process of removing the information within the domain of the bubbles is shown

569 in figure 12 . The red dots represent the resulting centroid location estimation

570 after removing the undesired bubbles induced information. It is clear that the

571 bubbles removal process drastically reduced the centroid location range from

572 about 15 pixels to about only 2 pixels.

573 It is important to mention that following the aforementioned procedure, we

574 can keep the bubbles induced uncertainty low even for high void fraction cases.

575 The only impact on the liquid velocity measurements at high void fractions

576 will be reflected on the sampling number. Only those particles which view is

577 not blocked by a bubble are considered. Therefore many more images will be

578 required to compute reliable statistics for high void fraction cases.

579

[Figure 12 about here.]

580 Figure 13 shows the centroid location RMS comparisons between three cases,

581 the unheated case, the boiling case with raw data, and the boiling case without

582 the bubbles unreliable information. The bubbles brought about a huge RMS 
583 on the order of 2.5 pixels which was reduced to about 0.4 pixels just after

584 removing the bubbles information. From the comparison of these two cases,

$\mathbf{5 8 5}$ it is clear that the bubbles removal correction extends to the first four points

586 (from left to right) of the profile. These points are closer to the heated wall. The

587 fifth and sixth points show a significant discrepancy compared to the unheated

588 case. This discrepancy may be explained by the density fluctuations effects or

589 by the distorting effect located on the vicinity of the bubbles interface. From

590 this information the maximum bubbles induced optical distortion for the near-

591 wall measurements accounts for about $\sigma_{T O D_{b}}=0.2$ pixels. This value does not

592 take into account the density fluctuation influence which accounts for about

5930.2 pixels, a similar value was obtained for the density fluctuations induced

594 uncertainty estimated in previous sections, which accounted for $\sigma_{T O D_{\rho}}=0.16$

595 pixels, therefore the assumption of a linear dependency of the density induced

596 uncertainty with respect to location seems to be reasonable.

598 4.3. Data Analysis Issues

599 4.3.1. PTV Related

600 The previous sections showed critical issues that have to be addressed when

${ }_{601}$ using PTV for the analysis of subcooled boiling experimental images. These

602 issues were mainly related to optical distortions due to density gradients and

${ }_{603}$ due to the existence of boiling bubbles in the measurement region. Based on this

604 information, it was decided that careful removal of bubbles induced vectors was

605 a necessity to ensure reliable liquid velocity measurements. The bubbles induced

606 vectors removal can be performed in two distinct ways: The first one, which will

${ }_{607}$ be called Bubble Image Masking (BIM), is an image processing algorithm that

${ }_{608}$ removes or masks the pixels that are within the domain of the bubbles. The

609 second one, which will be called Bubbles Velocity Masking (BVM), is a post-

610 processing algorithm that removes or masks the velocity vectors that are within

$6 \mathbf{6 1}$ the domain of the bubbles. In both cases, the bubble domain is determined

612 from the shadowgraphy images acquired simultaneously with the PTV images. 
${ }_{613}$ Figure 14 shows the comparison of the average liquid velocity profiles using three

614

615

616

618

619

620

621

622

623

624

625

626

627

628

629

630

631

632

633 4.3.2. Bubble intermittency

634

635

636

637

638

639

640

641

642 process.

\subsubsection{Bubble intermittency}

different PTV analysis procedures, including the Raw data analysis, on which no bubble information was removed, the PTV analysis on which BIM was used to remove undesired noise from bubbles images, and the PTV analysis with BVM, to remove undesired velocity vectors within the bubbles domain. These results are compared at different axial positions $(\mathrm{x}=0.0 \mathrm{~mm}, 1.2 \mathrm{~mm}$, and $6.2 \mathrm{~mm}$ ) from the nucleation site. From these results, it is clear that the PTV analysis with BIM produces an overestimation of the liquid velocity at the axial location $\mathrm{x}=6.2 \mathrm{~mm}$. Investigation of these results showed that the bubbles image removal of the BIM process hindered the particle centroid location of the PTV algorithm, resulting on spurious particles on the vicinity of masked regions. The spurious particles location corresponded to the bubbles interfaces, which lead to erroneously measure the bubbles velocities. This explains the overestimation of the liquid velocity at the location $\mathrm{x}=6.2 \mathrm{~mm}$, at this location the bubbles are fully developed and have larger velocities. Based on these results, it was determined that the BVM performed better, not only the spurious particles detection was reduced with the BVM process, but also provided higher quality vectors due to the aid of the bubbles information on the particle matching

Although it appeared that at this experimental condition a stable bubble nucleation behavior was achieved, the influence of the additional power supplied by the laser showed to be large. When performing PIV experiments in a continuous mode (see timing configuration explanation), the nucleation site behavior changed drastically (these effect were explained in previous sections). Therefore it was decided to perform the PIV experiments only in straddling mode configuration. In straddling mode, the laser induced power was largely reduced, and the effect of the laser in the nucleation behavior was not longer noticeable. However, some intermittency was observed in the activity of the 
643 nucleation site. As shown in Figure 15 this intermittency was detected directly

${ }_{644}$ from the experimental images. Ten percent of the 10,000 PIV images were con-

645 sidered as "intermittent" instances on which a lower void fraction was found.

${ }_{646}$ Examples of what was considered an "intermittent" image are shown in Figure

647 (A), (B), and (C). Intermittent images were removed from the analysis of

648 flow statistics because they were not representative of the "stable" behavior of

649 the nucleation site. Figure 15 (D) and (E) are examples of the experimental

650 data that was considered in the statistical analysis. As of today, is not known

651 with certainty the reasons of this intermittency.

652

653

654

655

656

657

658

659

660

661

662

663

664

665

666

667

668

669

670

671

[Figure 15 about here.]

Further insights are obtained when comparing the liquid velocity measurements between the stable case and the intermittent instances. Figure 16 shows the average velocity profiles at three axial positions $(\mathrm{x}=6.2, \mathrm{x}=1.19, \mathrm{x}=0.0)$ from the nucleation site. The black filled symbols represent the stable case or in the context of this condition, it represents instances of high void fraction on which the bubble number was larger than six, the red hollow circles represent the intermittent cases on which low void fraction was found. Low void fraction cases were those on which less than 6 bubbles were present. Comparing the results obtained between the intermittent and the stable cases, the influence of the bubbles parameters is clear. For the intermittent cases, the small void presence barely influence the liquid velocity, in fact the deviation from a single phase behavior was small. However for the stable case on which the bubble layer was fully developed, the influence of the high void fraction brought about a large acceleration of the liquid velocity.

\section{[Figure 16 about here.]}

\subsubsection{Sampling number influence on velocity measurements.}

To ensure that the measured flow statistics have been computed with a high statistical significance, an analysis was performed to test the influence of sampling number on the statistical parameters of interest. In this case we were 
672 interested on the wall-normal velocity profiles along the heater, to this end, the

673 velocity fields from the PIV images were divided in bins, 200 vertical bins in 674 the wall-parallel direction, and 50 horizontal bins in the wall-normal direction.

675 This allowed us to provide velocity profiles representing the flow development 676 along the heater.

677

For the uncertainty analysis, we focused on a single horizontal bin and per-

678 formed the average wall-normal velocity profile estimation depending on differ-

679 ent sampling numbers. The selected sampling numbers ranged from 10 to 4000 .

680 This analysis was repeated five times, ensuring that each time different samples

681 were selected out of the total sample pool which was about 4800 velocity fields.

${ }_{682}$ Figure 17 shows the results of this procedure. For small sample numbers, or in

${ }_{683}$ this case for small number of vector fields, the discrepancies of the five estimated

${ }_{684}$ velocity profiles are significant. These discrepancies gradually die down by in-

${ }_{685}$ creasing the number of vector fields, until reaching a point on which increasing

686 the number of vector fields no longer affects the estimated average profile. This

${ }_{687}$ point is said to have sufficient samples, enough to provide the velocity profile

${ }_{688}$ with high statistical significance. The point of high statistical significance for

689 the wall-normal velocity profile corresponded to a value of about 3000 vector

690 fields.

\section{Uncertainty Analysis}

${ }_{693}$ The uncertainty analysis on this work focus on the particle tracking velocime${ }_{694}$ try methodology. In this context, velocity measurements uncertainties strongly

695 depend on the accurate centroid location of the tracked objects (Kähler et al. 696 (2012)). PTV velocity measurements are obtained from individual particle lo${ }_{697}$ cations at different time intervals, and are given by $V_{i, x}=\left(x_{i, 2}-x_{i, 1}\right) / \Delta t_{P I V}$

698 where $V_{i, x}$ is the axial velocity component of the $i$-th particle, $x_{i, 2}$ is the axial ${ }_{699}$ position of particle $i$ in the second frame, while $x_{i, 1}$ is the axial position in the ${ }_{700}$ first frame, and $\Delta t_{P T V}$ is the time interval between two PTV frames. Similar 
expressions are required for the other velocity components, however, for sake of simplicity, only the axial component is treated. From this expression, and assuming that the timing uncertainty is negligible, it is clear that the centroid location uncertainty plays a key role on accurate velocity measurements. Two types of uncertainties were quantified, bias and random uncertainties. The next sections highlight the relevant aspects of these uncertainties.

\subsection{Bias uncertainties}

From the calibration grid experiments the bias uncertainty due to the thermal optical distortions ( $\left.\beta_{T O D}\right)$ was quantified. It was found that $\beta_{T O D}$ is a function of the heat flux, liquid velocity, and subcooling level, and that it remained constant for steady state conditions. For each test condition, bias uncertainties can be easily quantified in terms of a correction factor $\left(y_{o}\right)$. The correction of this bias becomes specially important for close to the wall measurements. Close to the wall velocity measurements are sensitive to image distortions which can induce significant uncertainties on the average and instantaneous velocities. The bias uncertainty had been addressed previously in optical or visualization studies dealing with strong density gradients and boiling (Roy et al. (2002); Hsieh et al. (2003)), however these studies did not addressed the fluctuating components of the uncertainties which are induced either by the random density fluctuations or by the bubbles influence, these are discussed next.

\subsection{Random uncertainties}

In previous sections, it has been found that the uncertainty associated with the centroid location estimation were those induced by thermally induced optical distortions (TOD), and those inherent of the PTV centroid location algorithm. Then, the centroid location uncertainties under boiling conditions can be expressed as $\sigma_{x}=\sigma_{T O D_{\rho}}+\sigma_{T O D_{b}}+\sigma_{P T V}$ (see section 4 for the definitions of each term). From the calibration grid experiments it was found that the uncertainty due to the random liquid density changes is a function of the position from the heated wall, with a maximum value at $y=0$ of $\sigma_{T O D_{\rho}}(0)=1.14$ pixels. The maximum uncertainty due to bubbles aberrations was found to be $\sigma_{T O D_{b}}=2.5$ 
pixels. Previous studies had shown that, for objects similar in size as the calibration grid points, the centroid location uncertainty of the PTV algorithm is $\sigma_{P T V}=0.05$ pixels $(\overline{\text { Estrada-Perez }}(2004) ;$ Estrada-Perez and Hassan $(2010)$.

In order to apply these results in our flow boiling experiments, a correction was required to account for the size discrepancies. The calibration grid points used for the uncertainty analysis accounted for about 25 pixels in diameter, while, in the flow boiling experiments, a maximum particle image size of about 2 pixels was found. Previous sensitivity studies had shown that the PTV centroid location performance is a strong function of particle size Estrada-Perez (2004); Estrada-Perez and Hassan (2010). After correcting for the size discrepancies, the maximum uncertainties suitable for the boiling experiments are: $\sigma_{T O D_{\rho}}(0)=$ 0.091 pixels, $\sigma_{T O D_{b}}=0.200$ pixels and $\sigma_{P T V}=0.004$ pixels, which adds up to a maximum centroid location uncertainty of $\sigma_{x}=0.295$ pixels. Using the calibration for the boiling experiments (a resolution of $17.5 \mu \mathrm{m} / \mathrm{pixel}$ ), $\sigma_{x}=5.17 \mu \mathrm{m}$. In terms of the axial velocity, the uncertainty propagation rules (Kähler et al. (2012); Kline and McClintock $(1953))$ gives $\sigma_{V_{x}}=\left(\sigma_{x_{1}}^{2} / \Delta_{t_{P I V}}^{2}+\sigma_{x_{2}}^{2} / \Delta_{t_{P I V}}^{2}\right)^{1 / 2}$, assuming that $\sigma_{x_{1}}=\sigma_{x_{2}}$ we end up with $\sigma_{V_{x}}=\left(2 \sigma_{x}^{2} / \Delta_{t_{P I V}}^{2}\right)^{1 / 2}=0.014 \mathrm{~m} / \mathrm{s}$, which is about $7 \%$ of the maximum axial velocity. As explained in section 4 the dominant uncertainty is induced by spurious particles and vectors due to the bubbles influence. The bubbles induced uncertainties are reduced by neglecting the information within the bubbles domain from the liquid velocity fields. The remaining bubbles induced uncertainty after the removal procedure was estimated to be $\sigma_{T O D_{b}}^{*}=0.016$ pixels, which gives $\sigma_{x}^{*}=0.111$ pixels or $\sigma_{x}^{*}=1.95$ $\mu \mathrm{m}$, which in terms of the velocity gives $\sigma_{V_{x}}^{*}=0.006 \mathrm{~m} / \mathrm{s}$ or $3 \%$ of the maximum axial velocity. This analysis provides insight of the maximum possible uncertainties, those which will happen close to the heater wall in a boiling experiment. Far from the heated wall, the effect of the density fluctuations and bubbles influences will be negligible. For sake of comparison the uncertainty with the bubbles effect and after these effects are reduced are depicted in figure 18 For these profiles, it was allowed for the bubbles influences to extend to the whole profile, instead of being confined to the near-wall region. This to 
include cases of higher void fractions, or cases that the bubbles are uniformly distributed through the channel, such as in the case of isothermal bubbly flows.

In figure 18 , the uncertainty before removing bubbles effects are depicted as black error bars, while the uncertainties after removing the bubbles effects are shown in red. It is important to mention that both, horizontal and vertical error bars were obtained with this analysis. Horizontal error bars for the position uncertainty, and vertical error bars for the velocity uncertainty. In Table 2 are summarized the uncertainties estimated in this study showing comparisons for the bubbles effect and object size scaling corrections.

[Figure 18 about here.]

[Table 2 about here.]

\section{Results}

Velocity Measurements

After addressing the issues found in section 4 to reduce uncertainty and to enhance the statistics reliability, we focused on the liquid and bubbles statistics. The velocity of both phases was obtained by means of particle tracking velocimetry (PTV). The PTV algorithm is able to track small objects such as the PIV particle tracers or large objects such as the boiling bubbles. The results of the tracking algorithm are shown in Figure 19. The picture in the left of Figure 19 shows an instantaneous velocity field on top of the shadowgraphy image, the right picture in Figure 19 shows the PTV results of the boiling bubbles.

[Figure 19 about here.]

Figure 19 shows the advantages of using the single nucleation site approach. This approach provides the capability of measuring at the same experimental condition, the flow development from a heated single phase to the transition to two-phase boiling flow. Furthermore, the bubbles dynamics can be accurately characterized at every point along the heater, this allows to explore the influence of multiple bubble dynamics conditions on the average liquid velocity behavior. 
790 From a set of instantaneous information, like the ones shown in figure 19 average

812

813

814

815

816 is studied in more detailed in following sections. 


\subsection{Bubbles Dynamics}

Figure 21 shows the high magnification shadowgraphy results (resolution of $4.82 \mu \mathrm{m})$. The high magnification results allowed a detailed examination of the average bubble behavior along the bubble path. Four profiles are shown in Figure 21 bubbles width, height, axial velocity, and normal velocity. It is important to note that more than 20000 bubbles were considered so as to show the most probables behavioral bubble paths along the heater wall. Furthermore, these results were obtained with the straddling timing mode, which provides an independent measuring sample for each image pair. As observed in Figure 21 large changes in the bubbles dimensions came about the first half millimeter after nucleation. Bubbles at the nucleation site increased rapidly both in width and height. The nucleated bubbles showed to follow two preferable growing paths, namely: Path A and Path B. Path A is characterized by a sharp increase on bubble size, following two typical behavior. The first behavior of bubbles following path $\mathrm{A}$ is a rapid bubble growth which causes bubble detachment. Most of the detached bubbles reduced in size and were pushed back to reattach to the wall. The second common behavior of bubbles that follow Path A, were those bubbles that coalesced with the leading bubble. The motion fluctuations induced by the coalescing process promoted as well bubble detachment. Bubbles that followed path B, consisted on nucleated bubbles that did not detach from the wall, and presented a smooth growing rate as they slid through the wall. The bubbles velocity was intimately related to the bubbles growing behavior. Several points are worth noting: First, the bubbles seems to have an almost constant acceleration at least in the first $3.5 \mathrm{~mm}$ after nucleation. However, the axial velocity within 0.25 to $0.75 \mathrm{~mm}$ showed to be largely fluctuating. This behavior is explained when considering that at this location, most of the coalescence, detachment and reattachment happens. Another interesting behavior is the normal bubbles velocity at the nucleation site. The force acting on the bubbles induced by the rapid growing at the nucleation site produced bubbles velocities, in the wall-normal direction, as large as $0.4 \mathrm{~m} / \mathrm{s}$. This velocity is large compared to the maximum axial velocity that was not larger than $0.25 \mathrm{~m} / \mathrm{s}$. After this 
849 very large velocity increase, the normal velocity tends rapidly to zero.

850

851 Insight into the benefits of performing multi-scale measurements can be 852 gained when comparing the results from Figure 21] and Figure 22. Figure 85322 shows the synchronized measurements at the same conditions as the ones 854 shown in 21 but from different view point and magnification. Figure 21 shows 855 the high-magnification side-view results, while in Figure 22 are shown the lows56 magnification front-view results. Figure 21 provides detailed bubble dynamics 857

858

859

860

861

862

863

864

865

866

867

868

869

870

871

872

873

874 6.2. Liquid Velocity Statistics

875

876

877

[Figure 21 about here.]

information, however this measurement is limited to only $3.5 \mathrm{~mm}$ from the nu-

cleation site. Figure 22 shows less detailed information but in a larger scale. To make the differences of scales between these measurements clearer, a small rectangle close to the nucleation site in Figure 22 depicts the measurement region from the side-view high-magnification camera. The first plot of Figure 22 shows

the evolution of the probable paths nucleated bubbles may take while sliding through the wall, a uniform dispersion is observed, similar to the spreading of a bubble plume. The second plot of Figure 22 shows the axial velocity of the bubbles, from this plot can be inferred that the terminal velocity of the bubbles is about $0.2 \mathrm{~m} / \mathrm{s}$. Bubbles reach to these velocities fairly rapidly, within the first $10 \mathrm{~mm}$ after nucleation. The velocity component on the direction parallel to the heater ( $\mathrm{Z}$ direction) is shown in the third plot of Figure 22, This velocity component is very close to zero, however there is a significant increase of this velocity with distance. Sliding bubbles increasing in size reach rapidly to a terminal axial velocity, and show to increase their zigzag motion in the direction parallel to the wall.

[Figure 22 about here.]

One of the main objectives of the PTV measurements is to obtain detailed information of the liquid velocity close to the heated wall under subcooled boiling conditions. Figure 23 shows the average axial velocity profile at different 
${ }_{878}$ axial locations. The color bar indicate the axial distance from the nucleation

879 site, starting with a blue color for locations on which only single phase flow is

880

881

882

883

884

885

886

887

888

889

890

891

892

893

894

895

896

897

898

899

900

901

902

903

904

905

906

907

found, the red color represents the location after $7 \mathrm{~mm}$ downstream the nucleation site. The influence of the boiling bubbles on the liquid velocity is clear.

There is a local peak in the region close to the wall. This local peak is related to the average bubble radius measured at each axial location.

[Figure 23 about here.]

To have a better understanding of the liquid behavior, it is always convenient to present these results in dimensionless form. The characteristic length and velocity used for the non-dimensionalization are $y^{+}=\left(y u_{\tau}\right) / \nu$ for the characteristic length and $u_{\tau}$ for the characteristic velocity, $u_{\tau}$ is the friction velocity defined as $u_{\tau}=\sqrt{\left(\tau_{w} / \rho\right)}$. The wall shear stress $\left(\tau_{w}\right)$ is obtained experimentally using the approximation for regions close to the wall $\tau_{w}=\mu d u /\left.d y\right|_{y=0}$. From the previous definitions we found a frictional velocity of $u_{\tau}=0.0125 \mathrm{~m} / \mathrm{s}$. It is important to note that the heated single phase friction velocity was considered as the characteristic velocity. Figure 24 shows the non-dimensional form utilizing the single phase heated frictional velocity $u_{\tau}=0.0125 \mathrm{~m} / \mathrm{s}$. From this plot, significant discrepancies from the law of the wall (continuous line in red) were observed even for the heated single phase cases. These discrepancies, rather than being experimental uncertainties, are attributed to the buoyancy driven flow induced in the vicinity of the heated wall. The single phase unheated experimental results have been shown (Estrada-Perez and Hassan, 2010) to match faithfully the law of the wall for locations above $y^{+}=1.0$ in a similar configuration. Also, the effect of the boiling level has been previously observed by Estrada-Perez and Hassan (2010). However, the present experiments focused in a single nucleation site, allowing to have different void fractions which depend on the location. Therefore, a single heat flux condition will provide different local void fractions, which simplified greatly the experimental and analysis work. In Figure 24 the red and gray lines represent the limits of the viscous sublayer and that of the buffer layer. Also, it is clear that the largest influence of the 
924

925

926

\footnotetext{
${ }^{2}$ Uncertainties quantified by means of artificial images and idealized characteristics
} 
935 two random uncertainties $\sigma_{T O D_{\rho}}$ and $\sigma_{T O D_{b}}$. The random uncertainties orig-

936

937

938

939

941

942

943

944

945

946

947

948

949

950

951

952

953 8. References

954 Abdelmessih, A., Hooper, F., Nangia, S., 1972. Flow effects on bubble growth 955 and collapse in surface boiling. International Journal of Heat and Mass Trans-

956

957

958

959

961

962 inate from random density fluctuations and due to the boiling bubbles. The bias uncertainty is easily corrected by means of side experiments involving a calibration grid, the uncertainty due to random density fluctuations $\sigma_{T O D_{\rho}}$ can not be easily corrected, but for the studied conditions did not largely affect the measurements.

It was also found that large uncertainties on the PTV measurements were induced by the boiling bubbles, therefore it is recommended to perform simultaneous PTV/Shadowgraphy experiments. The shadowgraphy experiments will dictate the bubbles domain, from which spurious velocity vectors can be removed to reduce this uncertainties.

The experimental methodology developed in this work, provided whole-field velocity fields of the two phases, bubbles dynamics quantification and heater wall temperature fields in a simultaneous manner with acceptable accuracy and repeatability. The data gathered using this methodology can be used as an experimental benchmark for validation of CFD codes and for the improvement and development of models and correlations for a better description of subcooled boiling flow.

fer $15,115-125$.

Barber, J., Brutin, D., Sefiane, K., Gardarein, J., Tadrist, L., 2011. Unsteadystate fluctuations analysis during bubble growth in a "rectangular" microchannel. International Journal of Heat and Mass Transfer 54, 4784-4795.

Chu, I.C., No, H.C., Song, C.H., 2011. Bubble lift-off diameter and nucleation

frequency in vertical subcooled boiling flow. Journal of nuclear science and technology 48, 936-949. 
${ }_{963}$ Dominguez-Ontiveros, E., Estrada-Perez, C., Ortiz-Villafuerte, J., Hassan, Y., 964 2006. Development of a wall shear stress integral measurement and analysis 965 system for two-phase flow boundary layers. Rev. of Scientific Inst. 77, 105103.

966 Elsinga, G.E., Oudheusden, B.W.v., Scarano, F., 2005. Evaluation of aero967 optical distortion effects in PIV. Experiments in Fluids 39, 246-256. doi 10. $968 \quad 1007 / \mathrm{s} 00348-005-1002-8$.

969 Estrada-Perez, C., 2004. Analysis, Comparison and Modification of Various 970 Particle Image Velocimetry (PIV) Algorithms. Master thesis. Texas A\&M 971 University. USA, Tx.

972 Estrada-Perez, C.E., Hassan, Y.A., 2010. PTV experiments of subcooled boiling 973 flow through a vertical rectangular channel. International Journal of Multi974 phase Flow 36, 691-706.

975 Gerardi, C., Buongiorno, J., Hu, L.w., McKrell, T., 2010. Study of bubble 976 growth in water pool boiling through synchronized, infrared thermometry 977 and high-speed video. International Journal of Heat and Mass Transfer 53, $978 \quad 4185-4192$.

979 Golobic, I., Petkovsek, J., Kenning, D., 2012. Bubble growth and horizontal 980 coalescence in saturated pool boiling on a titanium foil, investigated by high981 speed ir thermography. International Journal of Heat and Mass Transfer 55, $982 \quad 1385-1402$.

983 Hassan, Y., Gutierrez-Torres, C., Jimenez-Bernal, J., 2005. Temporal correla$\mathbf{9 8 4}$ tion modification by microbubbles injection in a channel flow. Int. Com. Heat 985 Mass Transfer 32, 1009-1015.

986 Hetsroni, G., Zakin, J., Lin, Z., Mosyak, A., Pancallo, E., Rozenblit, R., 2000.

987 The effect of surfactants on bubble growth, wall thermal patterns and heat 988 transfer in pool boiling. International Journal of Heat and Mass Transfer 44, $989 \quad 485-497$. 
Hsieh, S.S., Lai, W.C., Tsai, H.H., 2003. Ldv assisted bubble dynamic parameter measurements from two enhanced tubes boiling in saturated r-134a. International journal of heat and mass transfer 46, 4911-4923.

Kähler, C.J., Scharnowski, S., Cierpka, C., 2012. On the uncertainty of digital piv and ptv near walls. Experiments in fluids 52, 1641-1656.

Khan, A., 2012. Bubbly Flow Experiment in Channel Using an Optical Probe and Tracking Algorithm. Master thesis. Texas A\&M University.

Kim, T.H., Kommer, E., Dessiatoun, S., Kim, J., 2012. Measurement of twophase flow and heat transfer parameters using infrared thermometry. International Journal of Multiphase Flow 40, 56-67.

Klausner, J., Mei, R., Bernhard, D., Zeng, L., 1993. Vapor bubble departure in forced convection boiling. International journal of heat and mass transfer 36 , 651-662.

Kline, S., McClintock, F., 1953. Describing uncertainties in single-sample experiments. Mec. Eng. 75, 3-8.

Končar, B., Matkovič, M., 2012. Simulation of turbulent boiling flow in a vertical rectangular channel with one heated wall. Nuclear Engineering and Design $245,131-139$.

Koyasu, M., Tanaka, T., Sato, Y., Hishida, K., 2009. Turbulence structure of bubbly upward flow (high spatial and temporal resolution measurements using high speed time series ptv). Transactions of the Japan Society of Mechanical Engineers 75 part B, 1446-1453. Japanese language.

Lee, T., Park, G., Lee, D., 2002. Local flow characteristics of subcooled boiling flow of water in a vertical concentric annulus. Int. J. Heat Mass Transfer 28, $1351-1368$

Okawa, T., Ishida, T., Kataoka, I., Mori, M., 2005. On the rise paths of single vapor bubbles after the departure from nucleation sites in subcooled upflow boiling. International journal of heat and mass transfer 48, 4446-4459. 
1018

Ortiz-Villafuerte, J., Hassan, Y., 2006. Investigation of microbubble boundary layer using particle tracking velocimetry. J. Fluid Mechanics 128, 507.

Roy, R., Kang, S., Zarate, J., Laporta, A., 2002. Turbulent subcooled boiling flow experiments and simulations. J. Heat Transfer 124, 73.

Roy, R., Velidandla, V., Kalra, S., 1997. Velocity field in turbulent subcooled boiling flow. J. Heat Transfer 119, 754.

Situ, R., Hibiki, T., Sun, X., Mi, Y., Ishii, M., 2004. Flow structure of subcooled boiling flow in an internally heated annulus. Int. J. Heat Mass Transfer 47, $5351-5364$.

Takehara, K., Etoh, T., 1999. A study on particle identification in PTV-particle mask correlation method-. J. Visualization 1, 313-23.

Theofanous, T., Dinh, T.N., Tu, J., Dinh, A., 2002a. The boiling crisis phenomenon: Part ii: dryout dynamics and burnout. Experimental Thermal and Fluid Science 26, 793-810.

Theofanous, T., Tu, J., Dinh, A., Dinh, T.N., 2002b. The boiling crisis phenomenon: Part i: nucleation and nucleate boiling heat transfer. Experimental thermal and fluid science 26, 775-792.

Wang, Y., Sefiane, K., 2012. Effects of heat flux, vapour quality, channel hydraulic diameter on flow boiling heat transfer in variable aspect ratio microchannels using transparent heating. International Journal of Heat and Mass Transfer 55, 2235-2243.

Yun, B.J., Bae, B.U., Euh, D.J., Song, C.H., 2010. Experimental investigation of local two-phase flow parameters of a subcooled boiling flow in an annulus. Nuclear Engineering and Design 240, 3956-3966.

Yun, B.J., Park, G.C., Julia, J.E., Hibiki, T., 2008. Flow structure of subcooled boiling water flow in a subchannel of $3 \times 3$ rod bundles. Journal of Nuclear Science and Technology 45, 402-422. 
1045

\begin{tabular}{|c|c|c|}
\hline & & 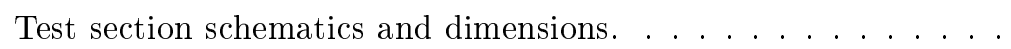 \\
\hline & 2 & isualization system schematics. \\
\hline & 3 & Timing configuration for semi-simultaneous PTV/shadowgraphy \\
\hline & & measurements, with square's color representing: green, illumina- \\
\hline & & tion by laser; gray, illumination by LED lamp; white, waiting or \\
\hline & & straddling period. \\
\hline & 4 & Bubble dynamics behavior at different illumination and timing \\
\hline & & conditions a) LED Straddling, b) LED Continuous, c) Laser+LED \\
\hline & & 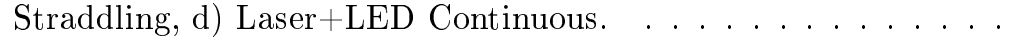 \\
\hline & 5 & Bubbles diameters comparison between the different illumination \\
\hline & & " and timing modes. . . . . \\
\hline & 6 & ure influence due to different illuminations and tim- \\
\hline & & ing conditions. . \\
\hline 59 & 7 & Centroid location process \\
\hline & 8 & Estimated location of grid points . . . . . . \\
\hline & 9 & Location uncertainty due to refractive index fluctuations . . . . \\
\hline & 10 & ent position displacement due to temperature driven re- \\
\hline 63 & & fractive index changes. . . . . . . . . . . . . . . . . . \\
\hline & 11 & le of centroid location variation due to a bubble optical \\
\hline & & distortion. . \\
\hline 66 & 2 & id location variation due to bubbles induced distortions. \\
\hline & $\overline{3}$ & stimation RMS variation due to bubbles in- \\
\hline & & tortions \\
\hline 69 & 14 & es effect on the average liquid velocity measurements \\
\hline 70 & 15 & ency of nucleation site activity. . \\
\hline & 16 & ency of nucleation site activity. . \\
\hline & 17 & Sampling number effect on the velocity profile. \\
\hline & 18 & ge velocity profiles compar- \\
\hline 74 & & s: a) with the bubbles effects (black error bars) and \\
\hline 75 & & ubbles effects (red error bars). \\
\hline 76 & 19 & Liquid and bubbles velocity fields and profiles. \\
\hline 77 & 20 & Void residence time and velocity profiles. \\
\hline 78 & 21 & Side view high magnification bubble dynamics measurments. . . \\
\hline & 22 & Low magnification shadowgraphy visualization for bubble dy- \\
\hline & & asurements . . . . . . . . . . . . . . \\
\hline & 23 & Axial liquid velocity profile at different axial positions from the \\
\hline & & nucleation site. \\
\hline & 2 & nal axial liquid velocity profile at different axial po- \\
\hline & & the single- phase friction \\
\hline & & red). \\
\hline
\end{tabular}




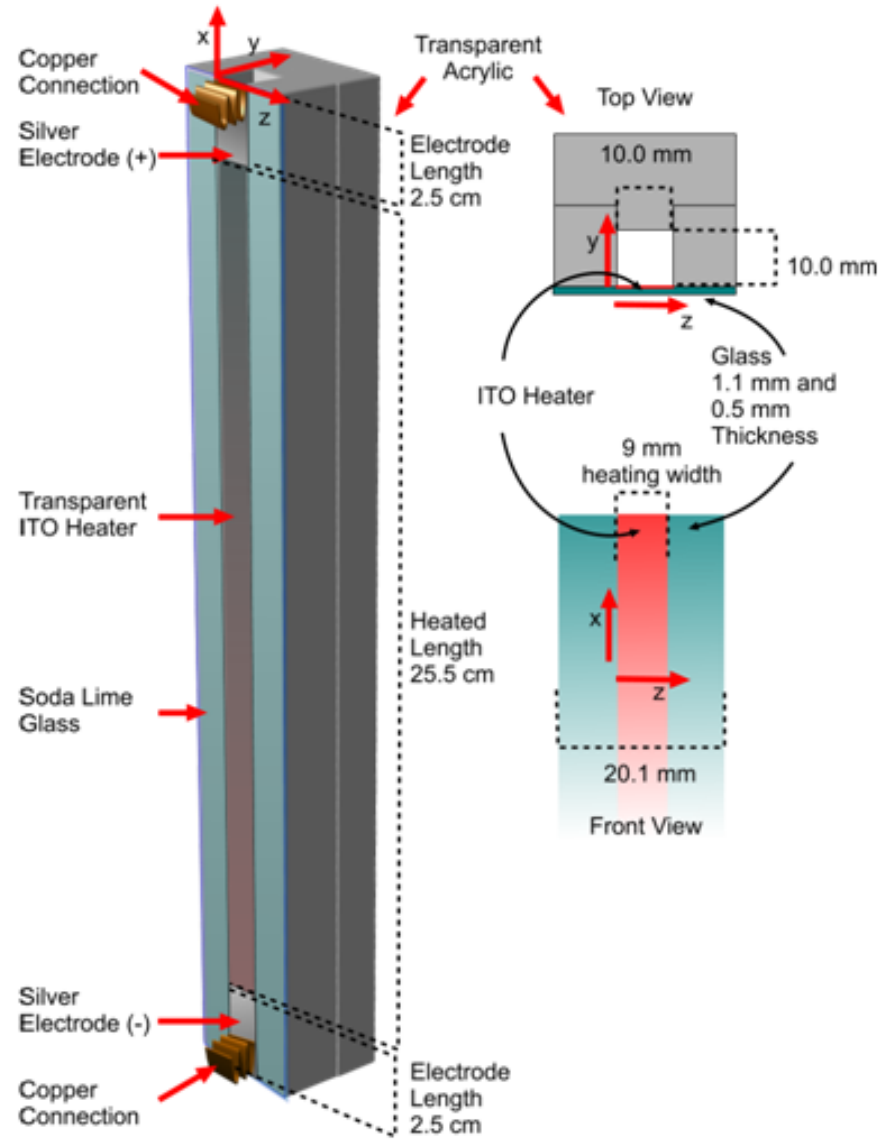

Figure 1: Test section schematics and dimensions. 


$$
e^{*}
$$




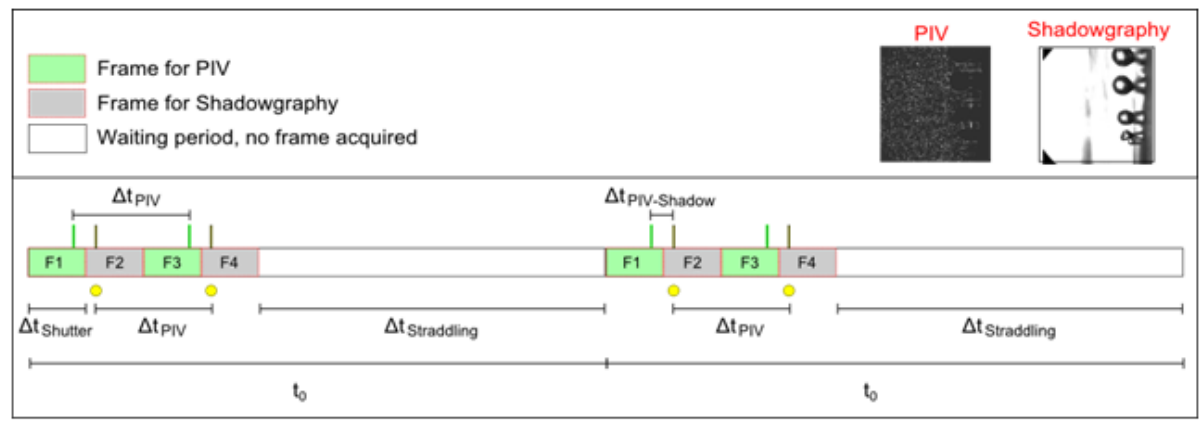

Figure 3: Timing configuration for semi-simultaneous PTV/shadowgraphy measurements, with square's color representing: green, illumination by laser; gray, illumination by LED lamp; white, waiting or straddling period. 


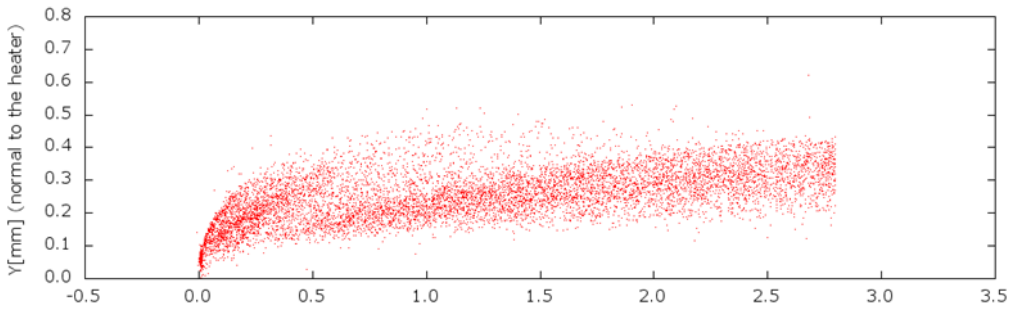

a)

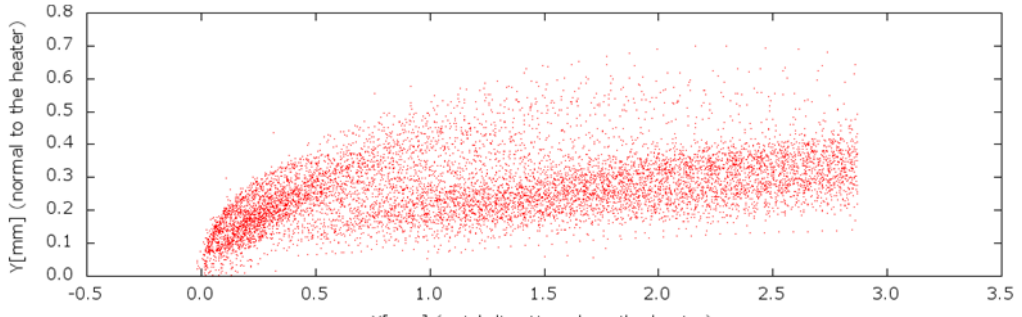

b)

X[mm] (axial direction along the heater)

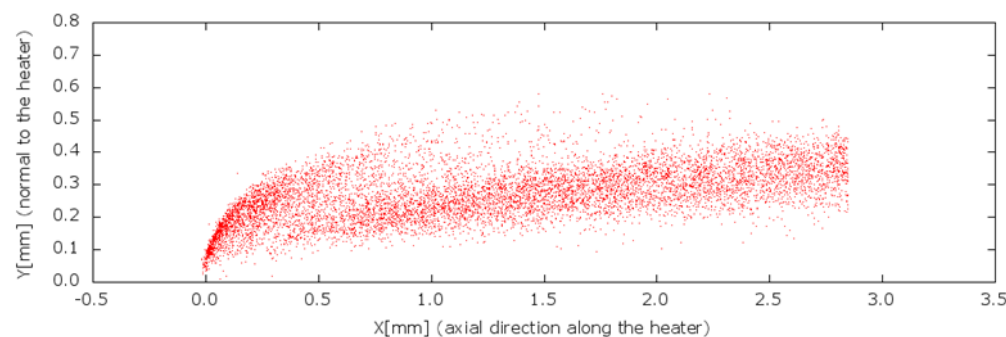

c)

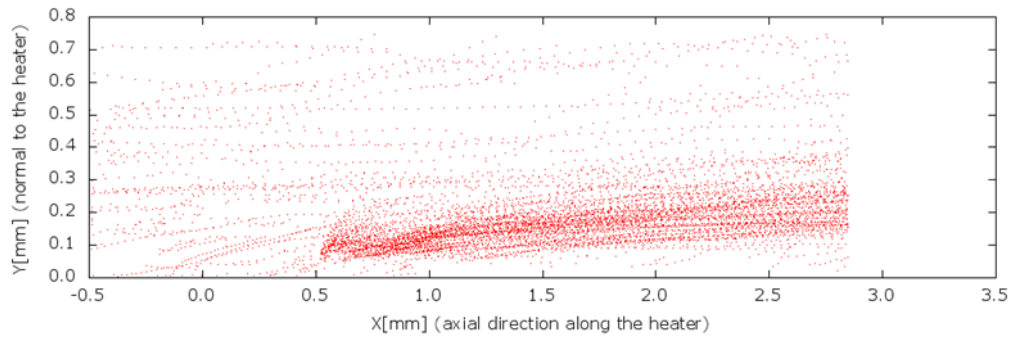

d)

Figure 4: Bubble dynamics behavior at different illumination and timing conditions a) LED Straddling, b) LED Continuous, c) Laser +LED Straddling, d) Laser+LED Continuous. 


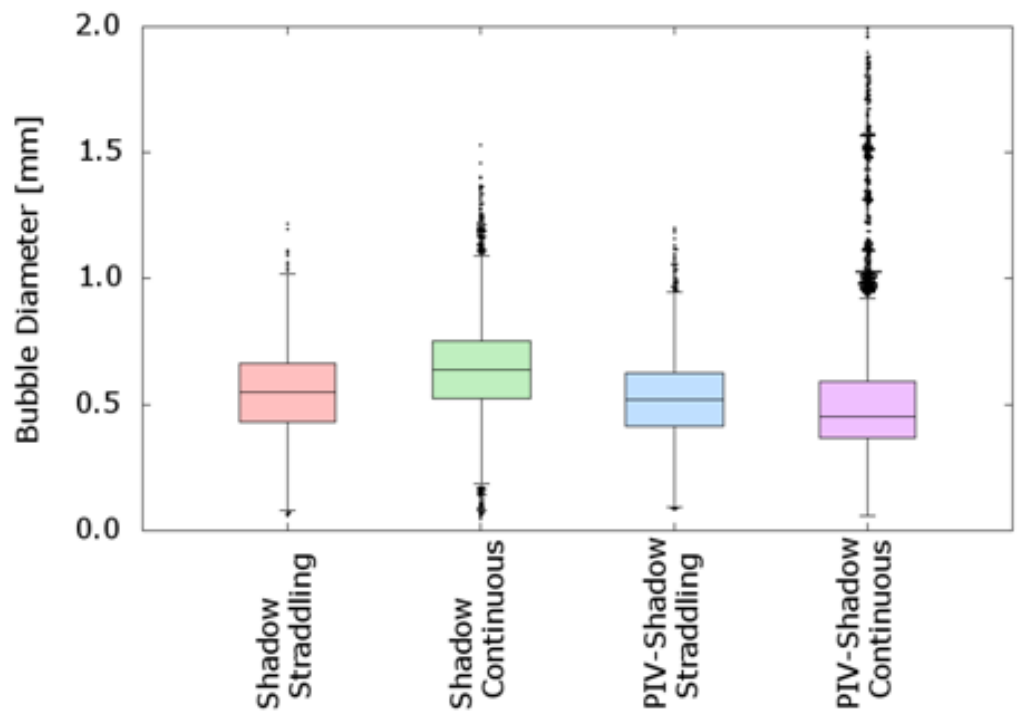

Figure 5: Bubbles diameters comparison between the different illumination and timing modes. 


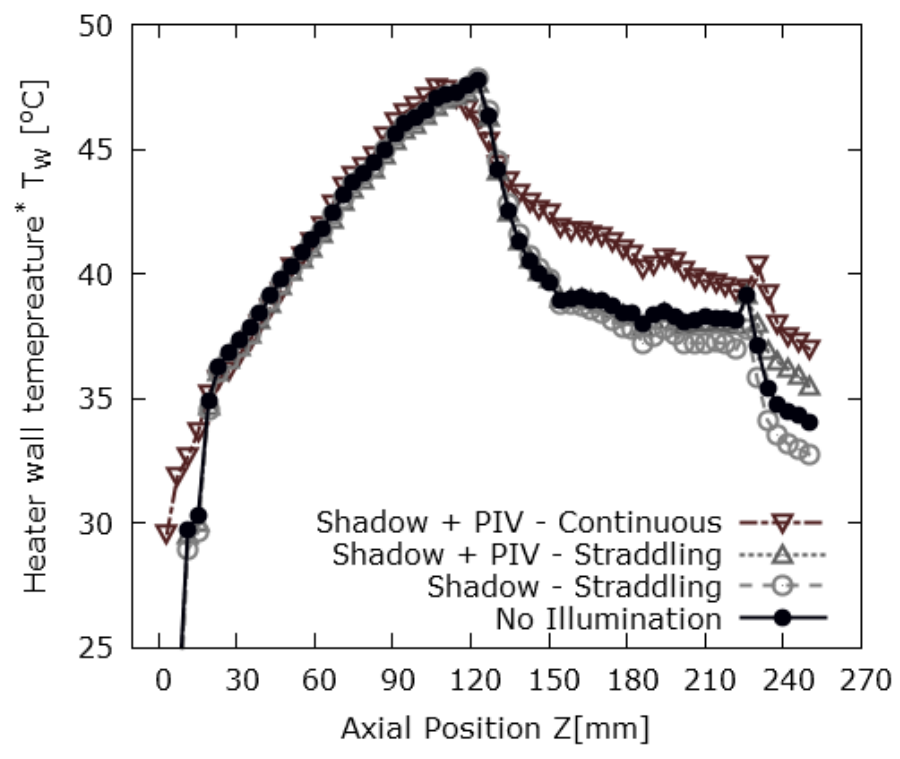

Figure 6: Wall temperature influence due to different illuminations and timing conditions. 


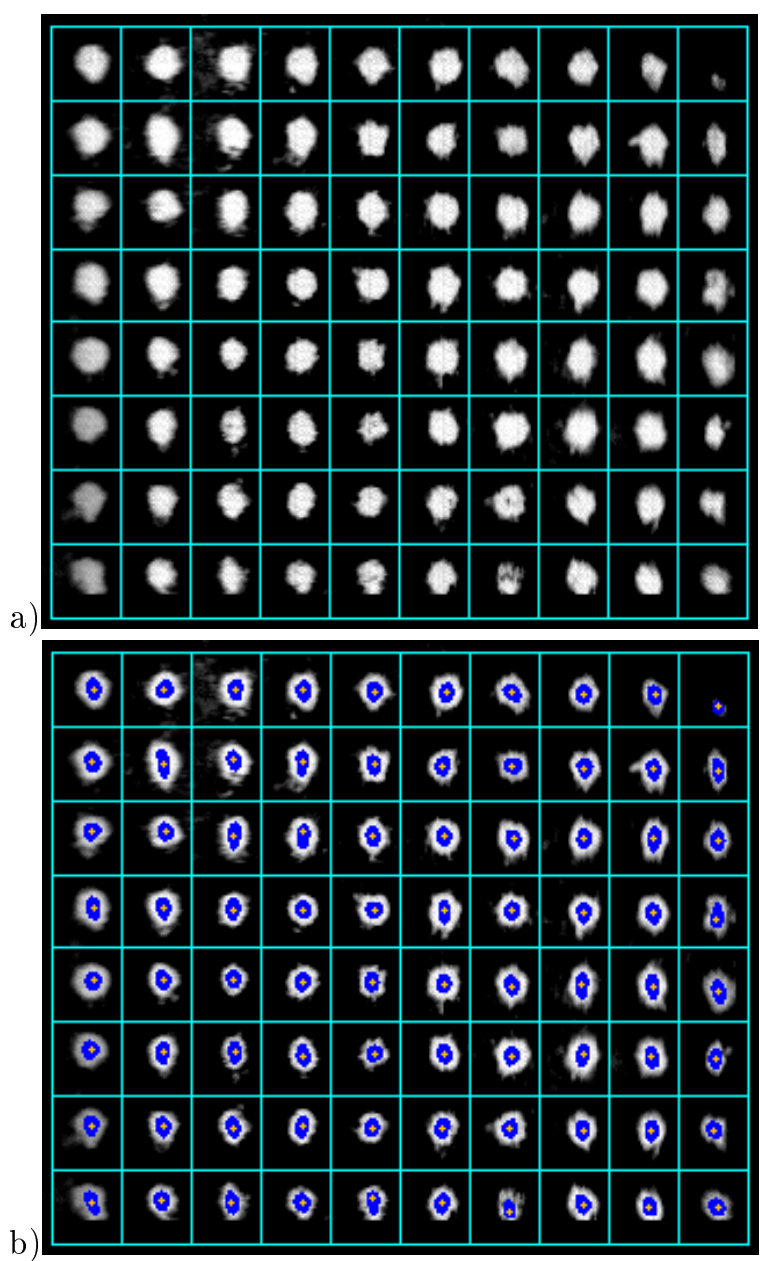

Figure 7: Centroid location process 


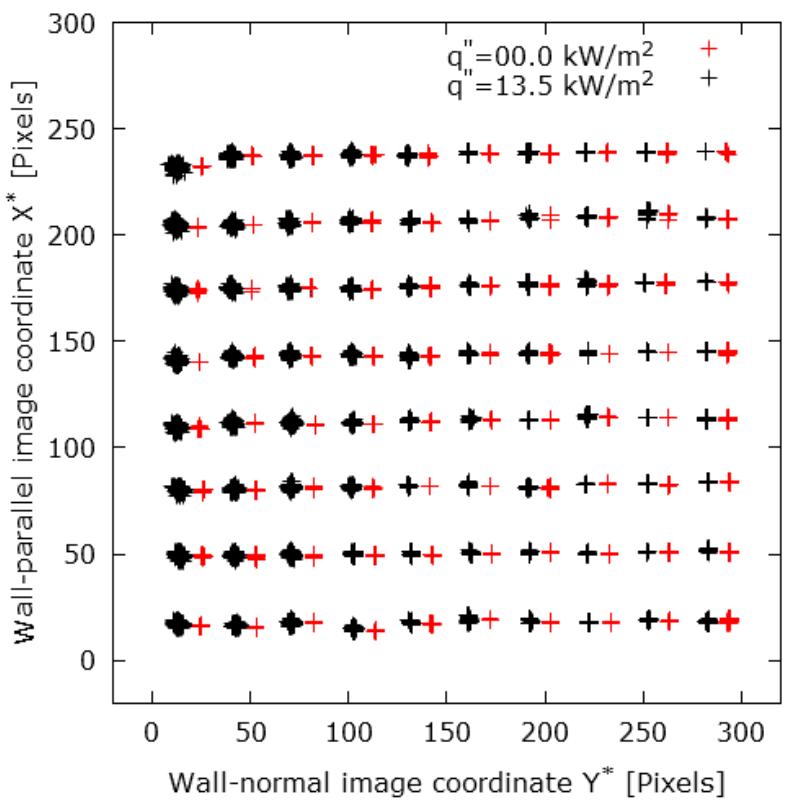

Figure 8: Estimated location of grid points 


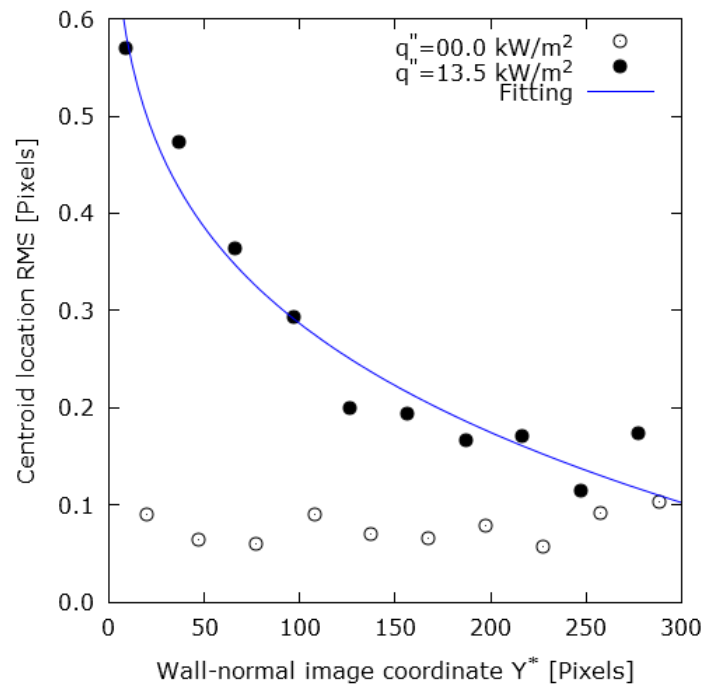

Figure 9: Location uncertainty due to refractive index fluctuations 


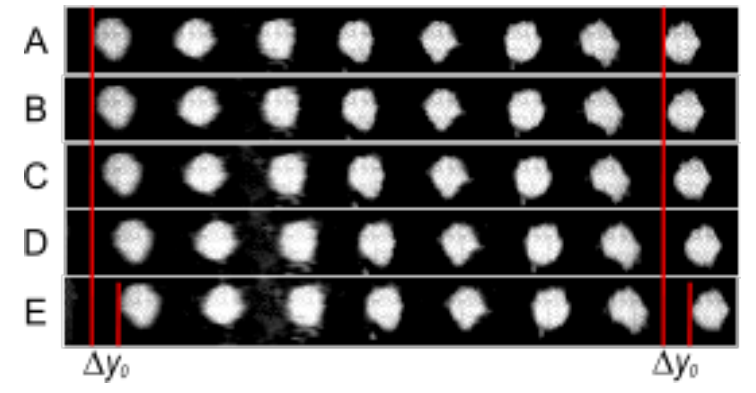

Figure 10: Apparent position displacement due to temperature driven refractive index changes. 
a) 00
oolos

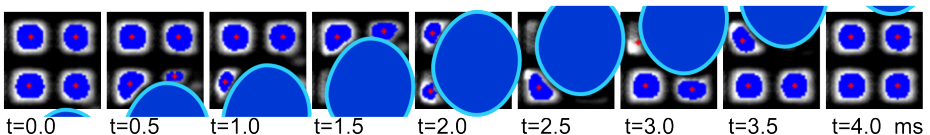

Figure 11: Example of centroid location variation due to a bubble optical distortion. 


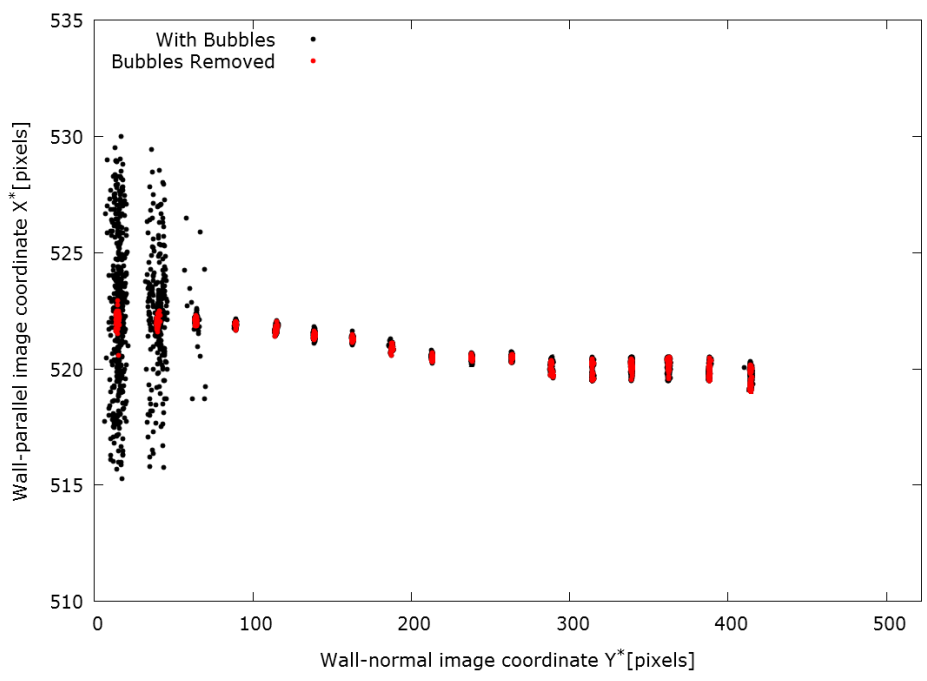

Figure 12: Centroid location variation due to bubbles induced distortions. 


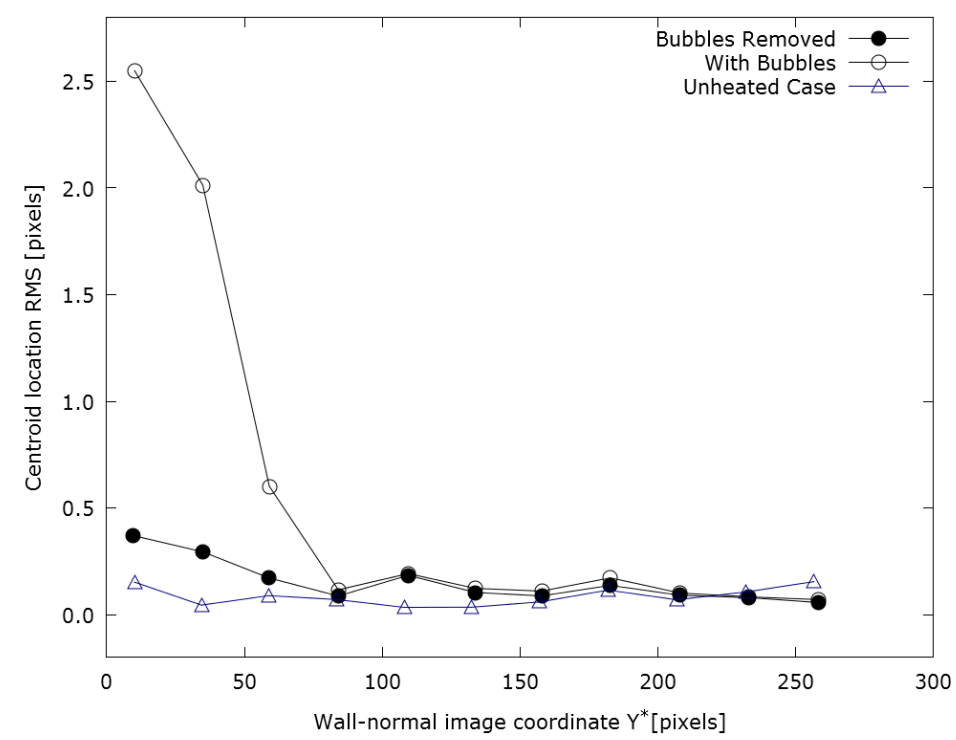

Figure 13: Centroid location estimation RMS variation due to bubbles induced distortions 


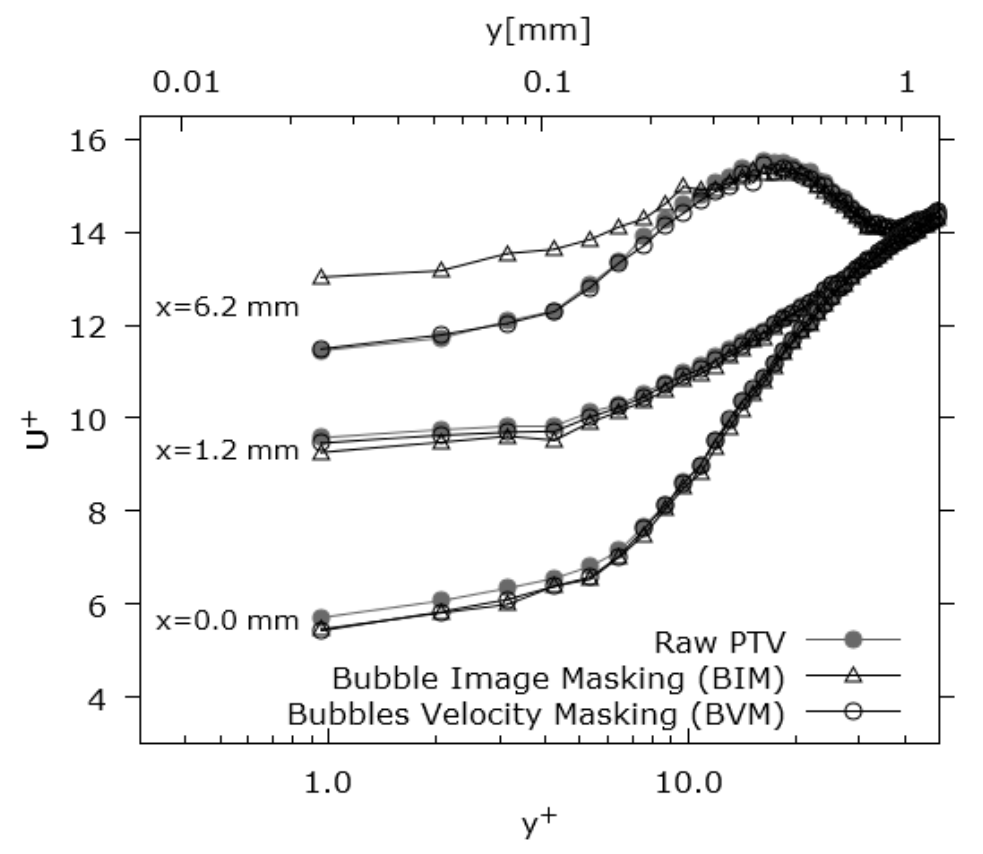

Figure 14: Bubbles images effect on the average liquid velocity measurements. 


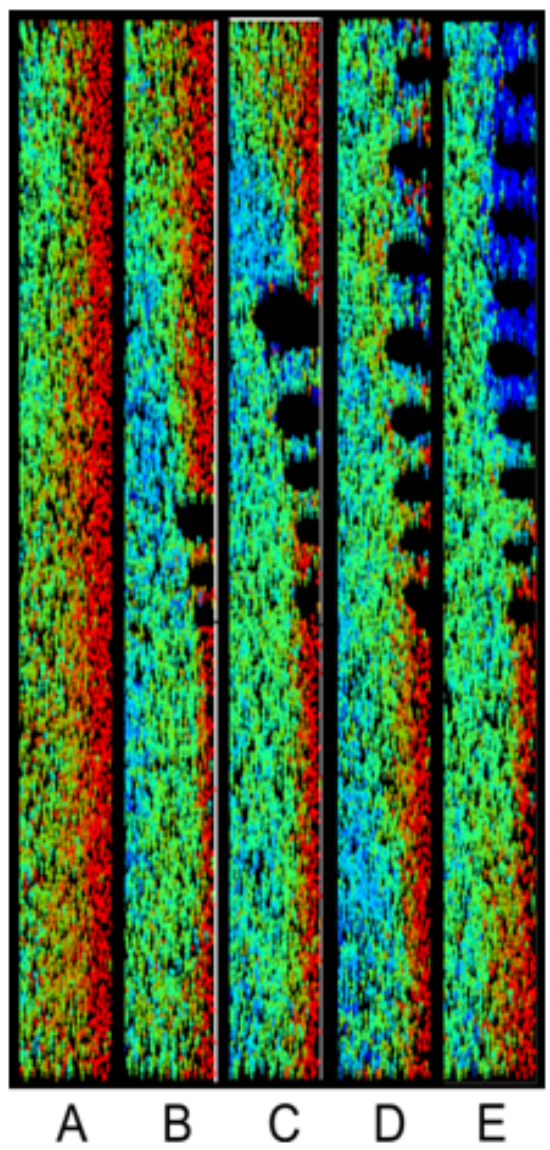

Figure 15: Intermittency of nucleation site activity. 


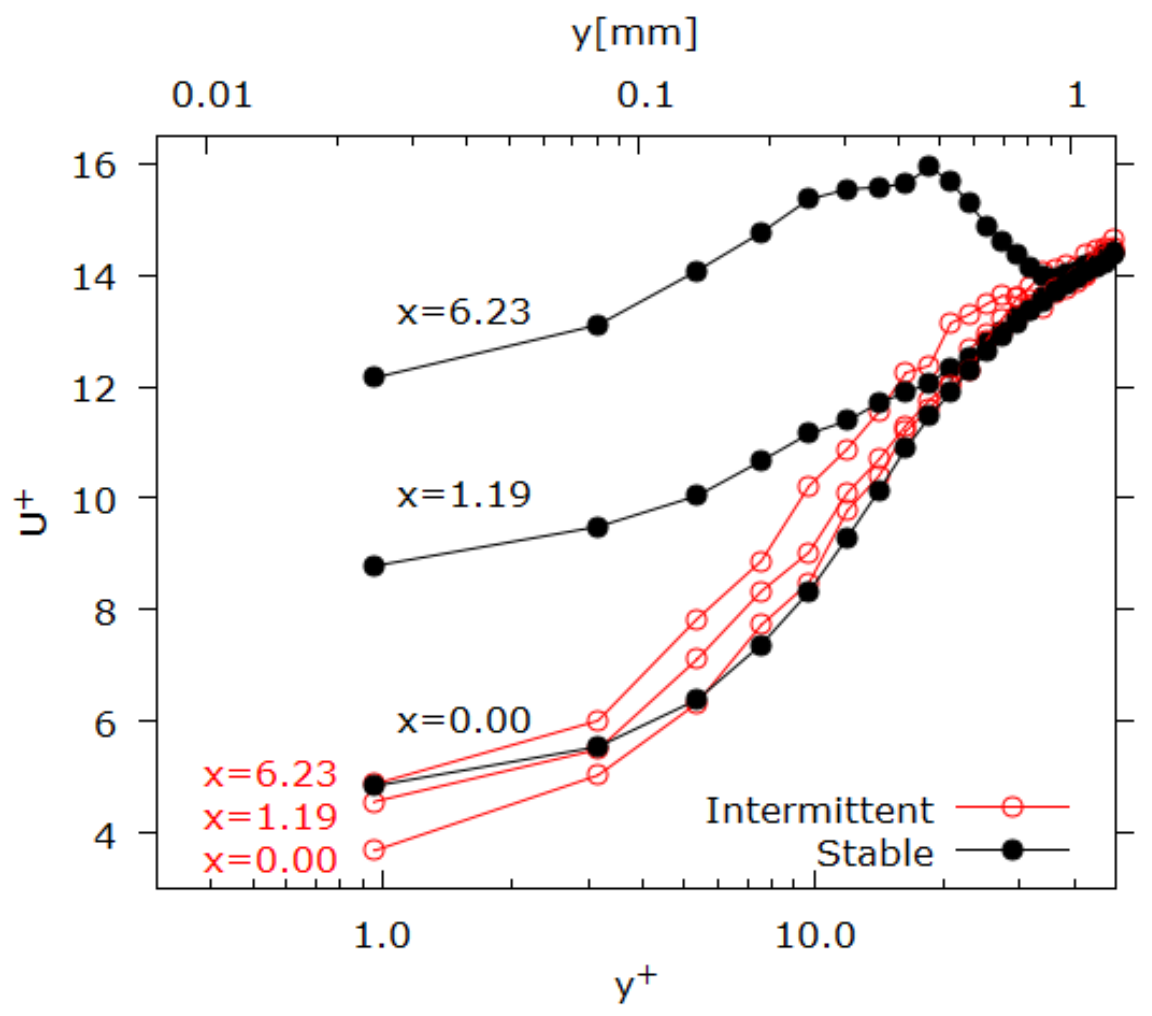

Figure 16: Intermittency of nucleation site activity. 


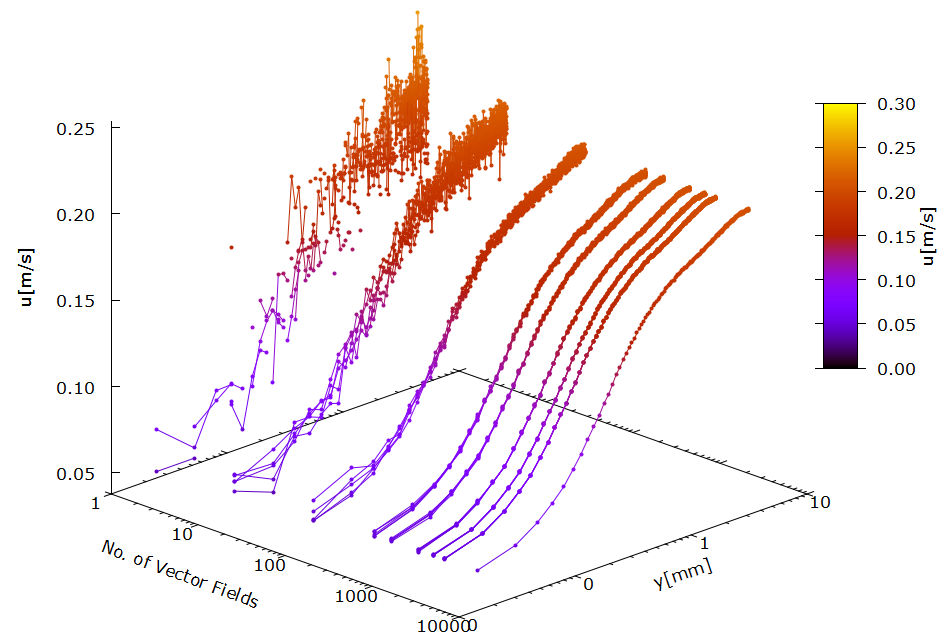

Figure 17: Sampling number effect on the velocity profile. 


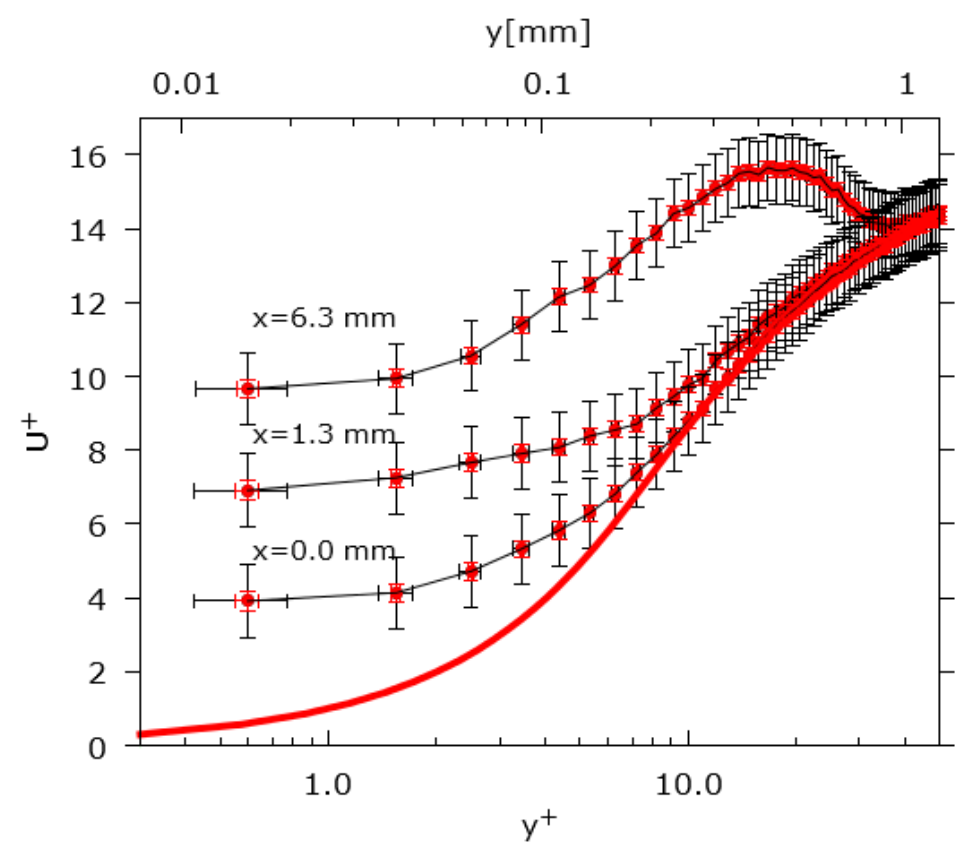

Figure 18: Uncertainty associated with the average velocity profiles comparing two cases: a) with the bubbles effects (black error bars) and without the bubbles effects (red error bars). 


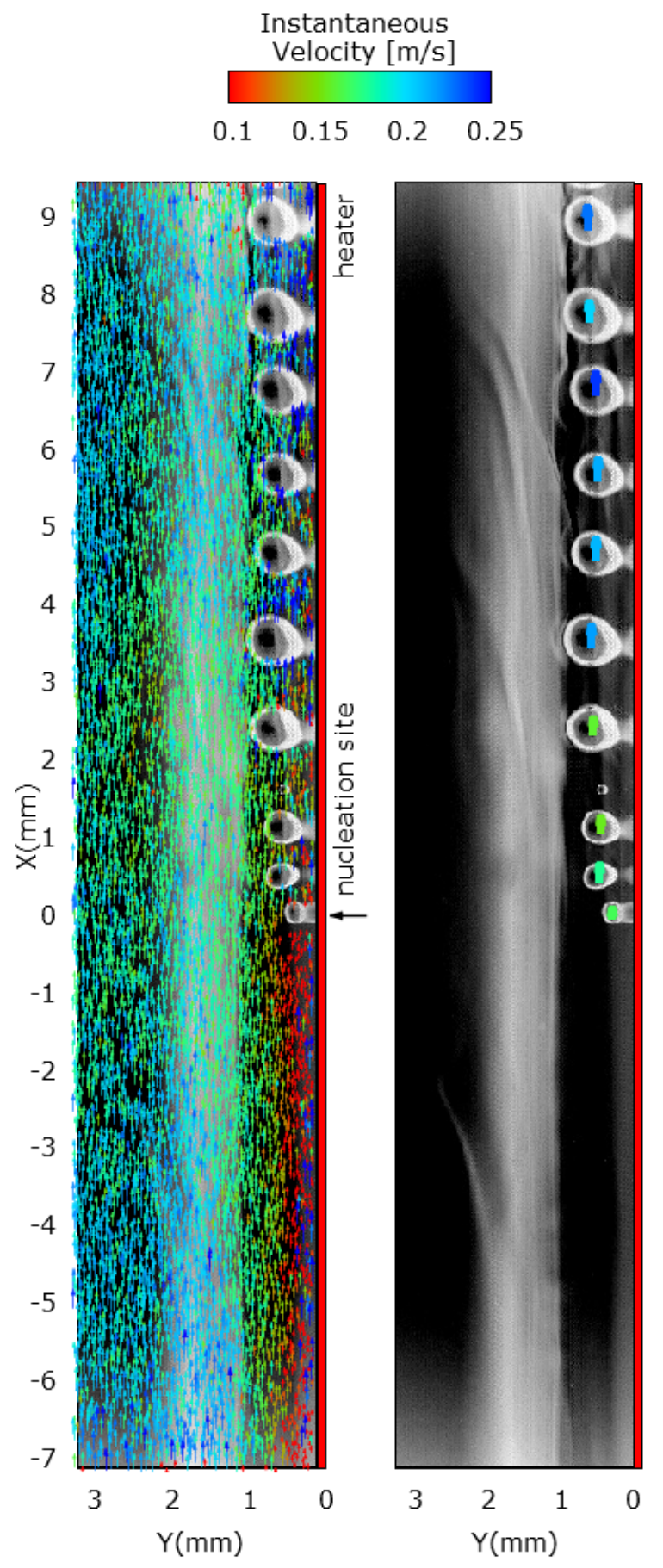

Figure 19: Liquid and bubbles velocity fields and profiles 


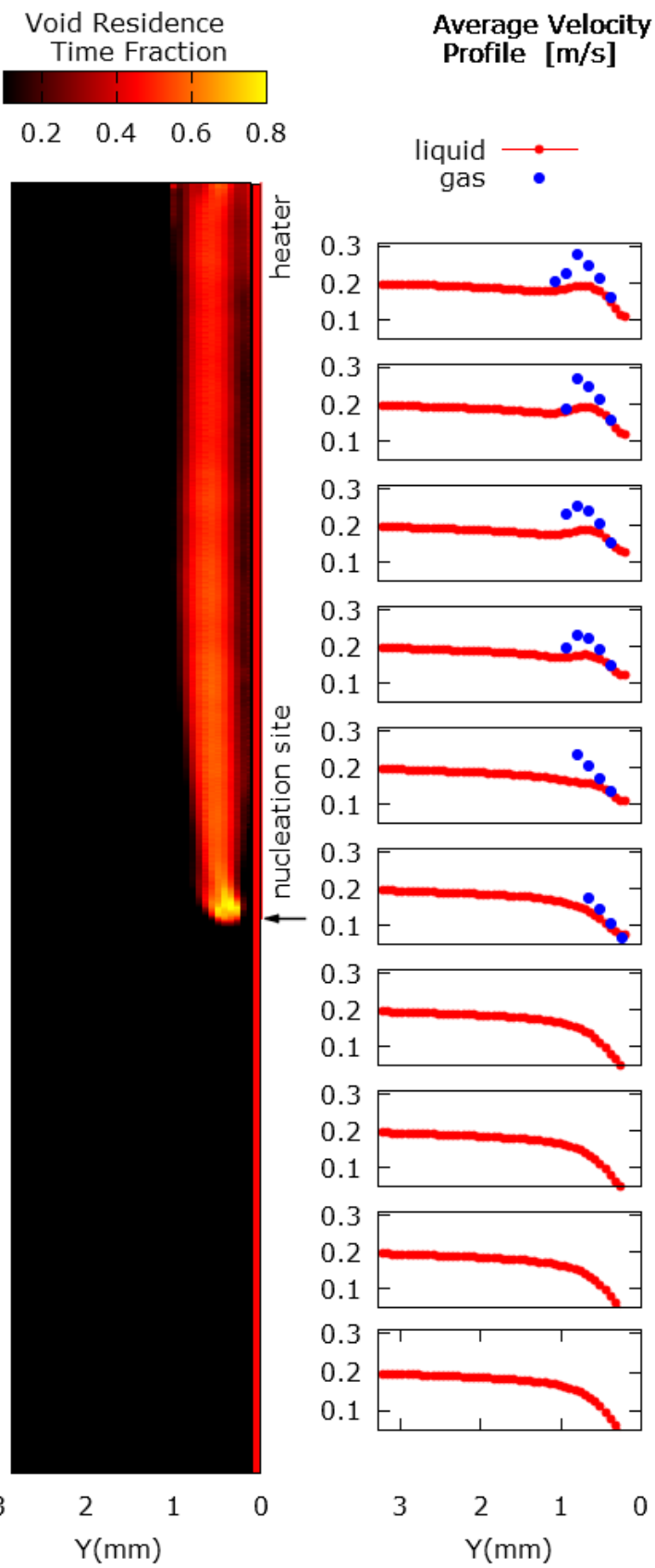

Figure 20: Void residence time and velocity profiles 

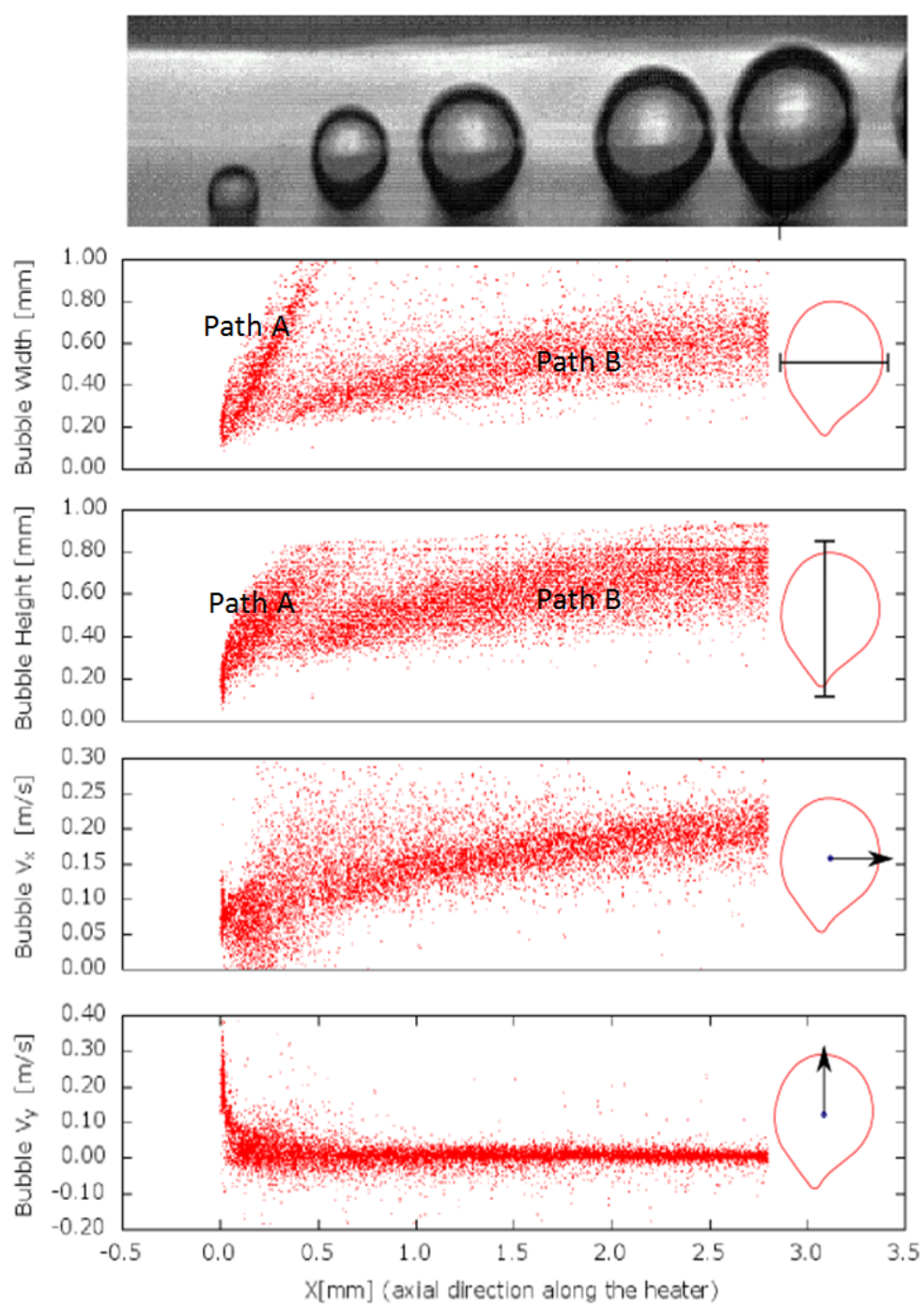

Figure 21: Side view high magnification bubble dynamics measurments. 


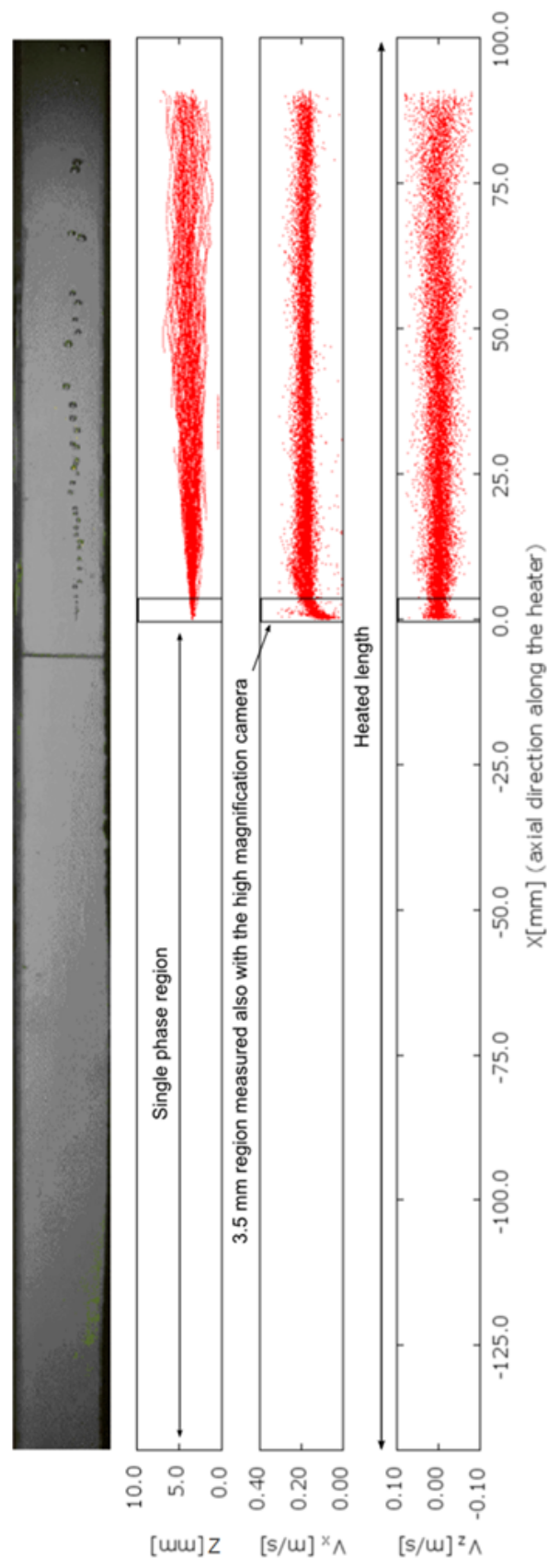

Figure 22: Low magnification shadowgraphy visualization for bubble dynamics measurements 


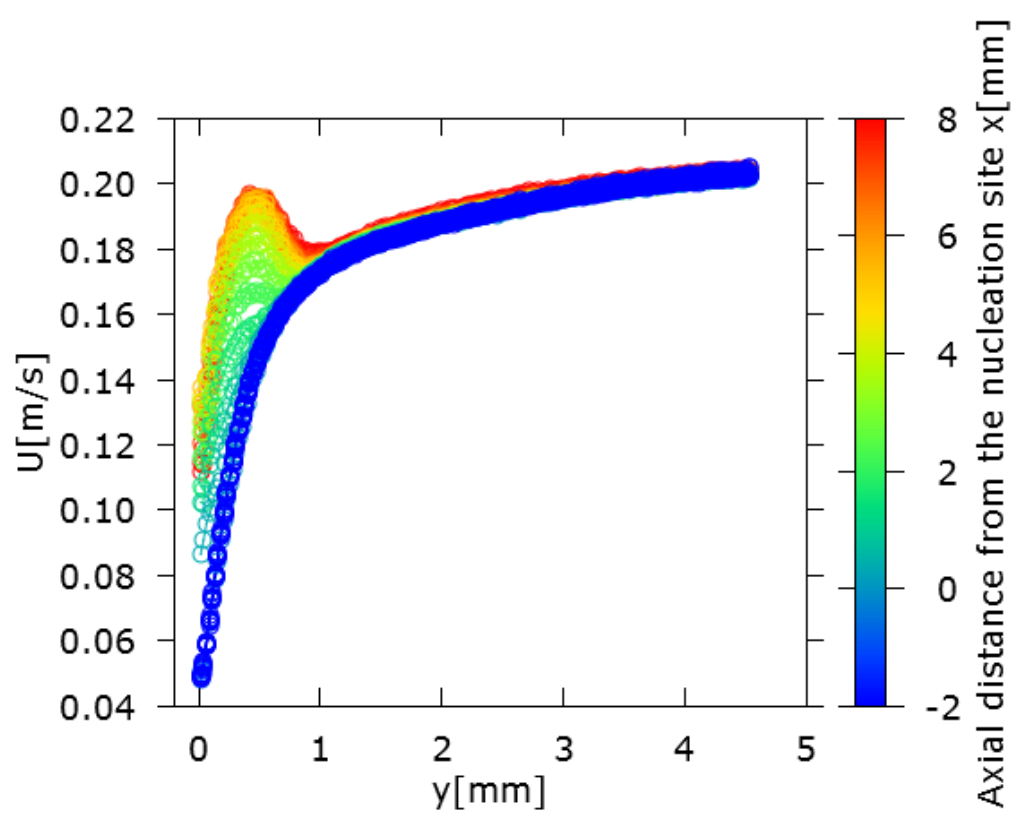

Figure 23: Axial liquid velocity profile at different axial positions from the nucleation site. 


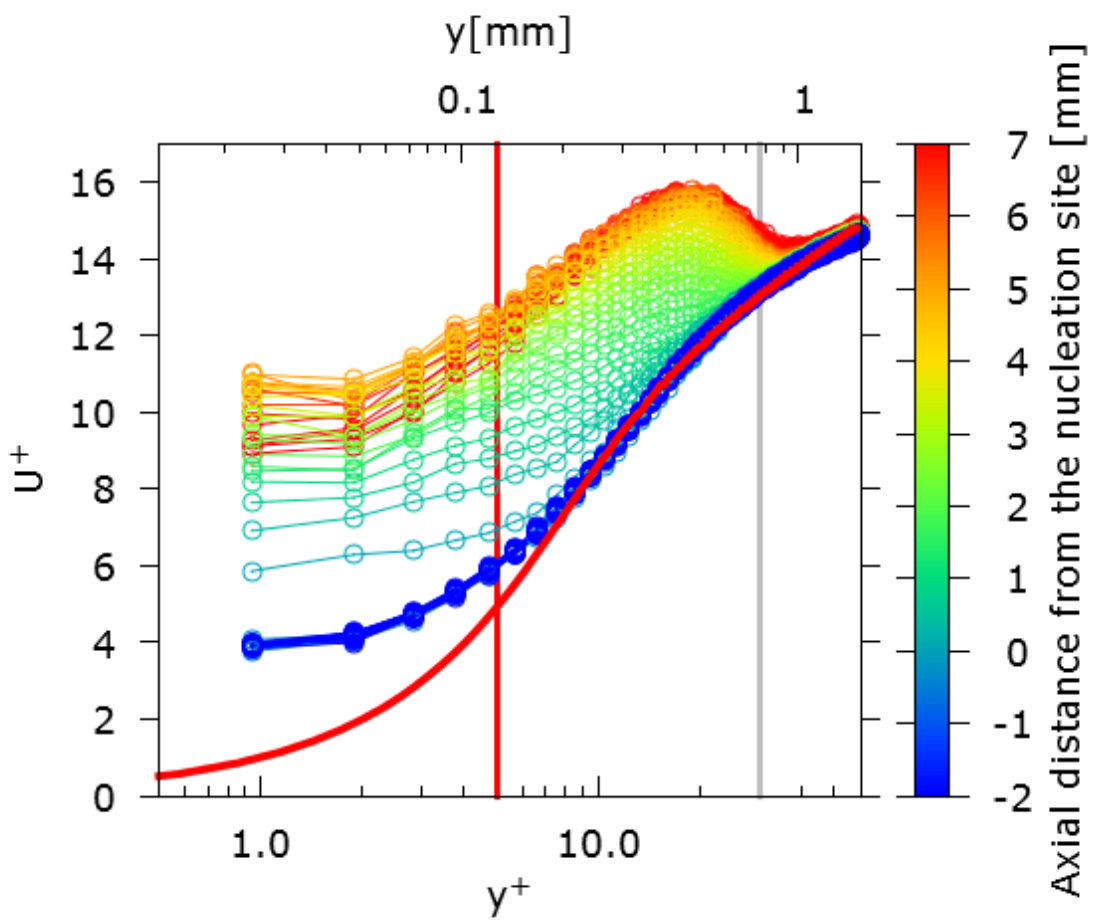

Figure 24: non-dimensional axial liquid velocity profile at different axial positions from the nucleation site (using the single- phase friction velocity $u^{*}$ ), and the single phase unheated law of the wall (red). 
1086 List of Tables

$1087 \quad 1 \quad$ Measurement equipment ranges and uncertainties . . . . . . . . 64

$1088 \quad 2 \quad$ Uncertainty Study Results . . . . . . . . . . . . 65 
Table 1: Measurement equipment ranges and uncertainties

\begin{tabular}{|c|c|c|}
\hline \multicolumn{2}{|l|}{ Parameter } & Uncertainty \\
\hline Liquid flow rate & {$\left[\mathrm{m}^{3} / \mathrm{s}\right]$} & $\dot{Q}_{l} \quad \pm 0.05 \%$ \\
\hline Temperature & {$\left[{ }^{\circ} \mathrm{C}\right]$} & $\begin{array}{l}T_{a m b} \pm 0.50 \\
T_{\text {in }} \pm 0.50 \\
T_{\text {out }} \pm 0.50 \\
\end{array}$ \\
\hline Differential Pressure & {$[\mathrm{Pa}]$} & $\begin{array}{ll}d P_{1} & \pm 0.25 \% \\
d P_{2} & \pm 0.50 \%\end{array}$ \\
\hline System Pressure & {$[\mathrm{Pa}]$} & $\begin{array}{l}P_{\text {in }} \pm 0.25 \% \\
P_{\text {out }} \pm 0.25 \% \\
\end{array}$ \\
\hline Heater power & {$[\mathrm{W}]$} & $\dot{q} \quad \pm 1.10 \%$ \\
\hline
\end{tabular}


Table 2: Uncertainty Study Results With bubbles effects

\begin{tabular}{c|c|c}
\hline \multirow{2}{*}{$\sigma_{T O D_{b}}$} & 2.50 pixels & 0.200 pixels \\
& $43.8 \mu \mathrm{m}$ & $3.5 \mu \mathrm{m}$ \\
\hline \multirow{2}{*}{$\sigma_{x}$} & 3.69 pixels & 0.295 pixels \\
& $64.6 \mu \mathrm{m}$ & $5.17 \mu \mathrm{m}$ \\
\hline \multirow{2}{*}{$\sigma_{V_{x}}$} & 5.21 pixels $/ \Delta t_{P I V}$ & 0.42 pixels $/ \Delta t_{P I V}$ \\
& $0.18 \mathrm{~m} / \mathrm{s}$ & $0.015 \mathrm{~m} / \mathrm{s}$ \\
\hline \multicolumn{3}{|c}{ Without bubbles effects } \\
\hline \multirow{2}{*}{$\sigma_{T O D_{b}}^{*}$} & 0.2 pixels & 0.016 pixels \\
& $3.5 \mu \mathrm{m}$ & $0.28 \mu \mathrm{m}$ \\
\hline \multirow{2}{*}{$\sigma_{x}^{*}$} & 1.39 pixels & 0.111 pixels \\
& $24.3 \mu \mathrm{m}$ & $1.95 \mu \mathrm{m}$ \\
\hline \multirow{2}{*}{$\sigma_{V_{x}}^{*}$} & 1.97 pixels $/ \Delta t_{P I V}$ & 0.16 pixels $/ \Delta t_{P I V}$ \\
& $0.069 \mathrm{~m} / \mathrm{s}$ & $0.006 \mathrm{~m} / \mathrm{s}$ \\
\hline \multicolumn{2}{c}{}
\end{tabular}

